\title{
Deep magnetic field stretching in numerical dynamos
}

\author{
Diego Peña ${ }^{1 *} \mathbb{D}$, Hagay Amit² and Katia J. Pinheiro ${ }^{1,2}$
}

\begin{abstract}
The process of magnetic field stretching transfers kinetic energy to magnetic energy and thereby maintains dynamos against ohmic dissipation. Stretching at depth may play an important role in shaping the field morphology and in the dynamo action. Here, we analyze snapshots from self-consistent 3D numerical dynamos to unravel the nature of field-flow interactions that induces stretching secular variation of the radial magnetic field at mid-depth of the shell. We search for roots of intense flux patches identified at the outer boundary. The deep radial field structures exhibit a position shift with respect to the locations of the outer boundary patches, consistent with a mixed effect of tangent cylinder rim and plume-like dynamics. A global stretching/advection rms ratio is 1.5-3 times larger than that of poloidal/toroidal flows. In addition, local stretching is often more effective than advection, in particular at regions of significant field-aligned flow. On average at roots of high-latitude flux patches, total stretching is 1.1 times larger than total advection despite the poloidal flow being only 0.37 of the toroidal flow. Radial stretching secular variation acts as an effective dynamo mechanism at regions where laterally varying radial flow shears toroidal field lines to generate a poloidal magnetic field. Stretching at depth exhibits similar parameter dependence as that of stretching at the outer boundary, with the strongest dependence being on the magnetic Prandtl number in both cases. Our results provide insights into the underlying deep dynamo mechanisms that sustain intense magnetic flux patches at the outer boundary.
\end{abstract}

Keywords: Magnetic field, Dynamo, Stretching, Flux patch, Secular variation, Core stratification

\section{Introduction}

The geomagnetic field is generated by rapidly rotating convective motions of an electrically conductive fluid in Earth's outer core. Temporal changes in the geomagnetic field termed secular variation (SV) may provide constraints on the fluid dynamics at the top of the core and possibly on the dynamo action. Indeed, geomagnetic field and SV models based on surface observations and satellite data (e.g. Jackson et al. 2000; Olsen and Mandea 2008) have been used to characterize Earth's core dynamics (e.g. Finlay and Jackson 2003), in particular the fluid flow at the top of the core (for a review, see Holme 2015), or as constraints on numerical dynamo simulations (e.g. Christensen et al. 1998, 2010; Aubert et al. 2013). In contrast, detailed morphological analyses of spatial patterns of the SV at depth have not been conducted.

\footnotetext{
*Correspondence: dpena@on.br

${ }^{1}$ Geophysics Department, Observatório Nacional, Rio de Janeiro 20921-400, Brazil

Full list of author information is available at the end of the article
}

According to the magnetic induction equation, the SV is comprised of magnetic advection, stretching, and diffusion. Magnetic field advection transfers magnetic energy from one degree to another, whereas magnetic field stretching transfers kinetic energy to magnetic energy and by that maintains dynamo action against ohmic dissipation (e.g. Moffatt 1978; Mininni 2011). Kageyama and Sato (1997a) found that in an $\alpha$-dynamo mechanism, axial convective cylinders generate a poloidal field from a basic toroidal field and vice versa (for illustration, see, e.g., Figure 5 of Olson et al. 1999). In these dynamo models, azimuthal magnetic field lines at the equatorial plane are transformed into a poloidal field in the form of axial field lines along the column, while axial magnetic field lines along the column are transformed into a toroidal field at the equatorial plane (see also Aubert et al. 2008b).

Morphological criteria for characterizing the observed geomagnetic field on the core-mantle boundary (CMB) include concentrated flux patches (Christensen et al. 2010). High-latitude normal flux patches (i.e., where the 
local field has the same sign as the axial dipole field) contribute significantly to the dominant axial dipole, while intensification and expansion of reversed flux patches (i.e., where the sign of the local field is opposite to that of the axial dipole field) diminish it (Gubbins 1987; Olson and Amit 2006). In addition, these patches have a particular signature on the SV. For example, an advected patch leads to a bipolar SV pattern (Livermore et al. 2017), whereas a patch intensified by downwelling gives a same-sign SV structure (Amit 2014). Due to the importance of these patches in defining the field morphology, it is essential to explore their origin. Local analysis of field-flow interactions may provide a detailed interpretation of the SV in the vicinity of these robust field features.

In regions of high-latitude intense geomagnetic flux patches, stretching may play an important role. These robust non-axisymmetric features are typically observed near the edge of the inner core tangent cylinder (Jackson et al. 2000), possibly due to a flow barrier and surface convergence at these regions (Olson et al. 1999). In dipole-dominated numerical dynamo models, surface convergence is correlated with columnar cyclones (Kageyama and Sato 1997b; Olson et al. 2002; Amit et al. 2007), so the flow near these patches has a large fieldaligned component and produces little magnetic advection (Finlay and Amit 2011). Magnetic field stretching may also be the underlying mechanism for regions of weak field intensity at Earth's surface. Strong deviations of the geomagnetic field from axial dipolarity appear in the form of reversed flux patches which may reflect expulsion of a toroidal field (Bloxham 1986) advected from depth to the CMB by fluid upwelling (e.g., Aubert et al. 2008b). The current weak surface field intensity in Brazil (Hartmann and Pacca 2009) is related to reversed flux patches on the CMB below the Atlantic (Aubert 2015; Tarduno et al. 2015), though this relation is not trivial (Terra-Nova et al. 2017).

The existence of stretching SV just below the CMB depends on whether a stably stratified layer is present (e.g., Whaler 1980). Stable stratification at the top of Earth's core might inhibit penetration of radial motion. Under such conditions, the tangential flow is purely toroidal. Some seismic studies (e.g., Helffrich and Kaneshima 2010) and mineral physics models (e.g., de Koker et al. 2012; Pozzo et al. 2012) suggest that the top of the core is indeed stably stratified (Gubbins and Davies 2013). In contrast, other studies claimed that the thermal conductivity of the core is as low as previously estimated (Konôpková et al. 2016; Ohta et al. 2016) and thus that the whole of the outer core convects. Even if the thermal conductivity is high, exsolution of mantle material (Badro et al. 2016; O'Rourke and Stevenson 2016) may destabilize the top of the core. Regional interpretations of the geomagnetic SV also suggest some local upwelling/downwelling
(Olson and Aurnou 1999; Chulliat et al. 2010; Amit 2014). Quasi-geostrophic core flow models rely on surface poloidal flow to infer the flow at depth (Pais and Jault 2008; Gillet et al. 2009, 2011, 2015). Lesur et al. (2015) argued that the geomagnetic data could not be adequately explained by a purely toroidal flow, but inclusion of a weak poloidal flow is sufficient to explain the SV, suggesting that the upper part of the core is weakly stratified.

Peña et al. (2016) studied global and local stretching at the top of the shell of numerical dynamos in detail. They found that stretching has a significant influence on the SV despite the relatively weak poloidal flow. In addition, their analysis showed that local stretching is often more effective than advection, in particular at regions of significant field-aligned flow as well as in intensifying magnetic flux patches. Morphological resemblance between local stretching in the dynamo models of Peña et al. (2016) and local observed geomagnetic SV (Amit 2014) may indicate the presence of stretching at the top of the Earth's core.

Of course dynamo action might not necessarily occur in the entire outer core. The dynamo may be generated exclusively at depth due to stable stratification at the top of the core. For example, the very weak, large-scale, and axisymmetric magnetic field of Mercury (Anderson et al. 2011; Oliveira et al. 2015) can be explained by a skin effect across a stably stratified layer (Christensen 2006; Christensen and Wicht 2008; Wicht and Heyner 2014). As mentioned above, such a layer was also proposed for the Earth (Pozzo et al. 2012; Gubbins and Davies 2013). In this paper, we analyze output from the same set of numerical dynamos as Peña et al. (2016), but deep in the shell, to understand the field-flow interactions and the contribution of magnetic field stretching to the SV there. In particular, we search for the roots of the intense magnetic flux patches, identified at the outer boundary by Peña et al. (2016), to explore their kinematic origins. We focus on the contribution of stretching as well as other mechanisms to the SV of the radial component of the field for comparison with the radial field on the outer boundary, which is the component accessible from observations. The contributions of the different SV mechanisms at depth and their dependence on the dynamo control parameters are explored. The results for the SV of the radial magnetic field at depth are discussed in the context of inductive effects and the dynamo process.

As mentioned above, the mechanism of magnetic field generation by convection of an electrically conductive fluid in a rotating spherical shell has been extensively studied using numerical dynamos (e.g., Kageyama and Sato 1997a; Olson et al. 1999; Aubert et al. 2008b; Takahashi and Shimizu 2012). Here, we focus on analyzing exclusively the different terms of the radial component of the magnetic induction equation and only at a specific spherical surface situated at depth. For practical reasons, 
we sample the deep shell only at mid-depth. We compare our findings at depth with the corresponding results just below the outer boundary (Peña et al. 2016).

The paper is outlined as follows. In the "Theory" section, we describe the theoretical basis of our study, including the different terms in the radial induction equation at depth, the effect of the tangent cylinder at depth, and an assessment of the image distortion effects from the CMB to mid-depth. The methods are described in the "Methods/Experimental" section, including numerical dynamos, the criterion for identifying roots of outer boundary patches, and the statistical tools used to evaluate the results. In the "Results" section, we report the global and local stretching contributions as well as their dependence on the dynamo control parameters. We discuss our main findings in the "Discussion" section.

\section{Methods/Experimental}

\section{Theory}

Radial magnetic induction equation far from the boundaries

The magnetic induction equation in a $3 \mathrm{D}$ vectorial form is given by

$$
\frac{\partial \vec{B}}{\partial t}=\nabla \times(\vec{u} \times \vec{B})+\lambda \nabla^{2} \vec{B}
$$

where $\vec{B}$ is the magnetic field, $\vec{u}$ is the fluid velocity, and $\lambda$ is the magnetic diffusivity. For comparison with information accessible from observations, we focus on the non-diffusive (or frozen-flux, denoted by superscript "ff") part of (1):

$$
\frac{\partial \vec{B}^{f f}}{\partial t}=\nabla \times(\vec{u} \times \vec{B})
$$

Using a vector identity for the right-hand side of (2) and considering the non-divergence of the flow and the field,

(2) can be rewritten as

$$
\frac{\partial \vec{B}^{f f}}{\partial t}=(\vec{B} \cdot \nabla) \vec{u}-(\vec{u} \cdot \nabla) \vec{B}
$$

We now consider the radial component of (3):

$$
\hat{r} \cdot \frac{\partial \vec{B}^{f f}}{\partial t}=\hat{r} \cdot(\vec{B} \cdot \nabla) \vec{u}-\hat{r} \cdot(\vec{u} \cdot \nabla) \vec{B}
$$

The first term of (4) is simply

$$
\hat{r} \cdot \frac{\partial \vec{B}^{f f}}{\partial t}=\frac{\partial B_{r} f f}{\partial t}
$$

where the subscript $r$ denotes the radial component. The second term of (4) is

$$
\hat{r} \cdot(\vec{B} \cdot \nabla) \vec{u}=B_{r} \frac{\partial u_{r}}{\partial r}+\frac{B_{\theta}}{r} \frac{\partial u_{r}}{\partial \theta}+\frac{B_{\varphi}}{r \sin \theta} \frac{\partial u_{r}}{\partial \varphi}-\frac{B_{\theta} u_{\theta}}{r}-\frac{B_{\varphi} u_{\varphi}}{r}
$$

where $\theta$ and $\varphi$ are colatitude and longitude, respectively. Considering $\nabla \cdot \vec{u}=0$

$$
\frac{\partial u_{r}}{\partial r}=-\nabla_{h} \cdot \vec{u}_{h}-\frac{2}{r} u_{r}
$$

and substituting (7) into (6) gives

$$
\hat{r} \cdot(\vec{B} \cdot \nabla) \vec{u}=-B_{r} \nabla_{h} \cdot \vec{u}_{h}+\vec{B}_{h} \cdot \nabla_{h} u_{r}-2 \frac{B_{r} u_{r}}{r}-\frac{B_{\theta} u_{\theta}}{r}-\frac{B_{\varphi} u_{\varphi}}{r}
$$

where the subscript $h$ denotes the component tangential to a spherical surface of constant radial distance. The third term of (4) is

$$
\begin{aligned}
\hat{r} \cdot(\vec{u} \cdot \nabla) \vec{B} & =u_{r} \frac{\partial B_{r}}{\partial r}+\frac{u_{\theta}}{r} \frac{\partial B_{r}}{\partial \theta}+\frac{u_{\varphi}}{r \sin \theta} \frac{\partial B_{r}}{\partial \varphi}-\frac{B_{\theta} u_{\theta}}{r}-\frac{B_{\varphi} u_{\varphi}}{r} \\
& =\vec{u}_{h} \cdot \nabla_{h} B_{r}+u_{r} \frac{\partial B_{r}}{\partial r}-\frac{B_{\theta} u_{\theta}}{r}-\frac{B_{\varphi} u_{\varphi}}{r}
\end{aligned}
$$

Finally, by substituting (5), (8), and (9) into (4), the radial component of the magnetic induction equation at a radius $r$ far from the boundaries, i.e., without assuming $u_{r}=0$, can be written as

$$
{\frac{\partial B_{r}}{\partial t}}^{f f}=-\vec{u}_{h} \cdot \nabla_{h} B_{r}-B_{r} \nabla_{h} \cdot \vec{u}_{h}-\frac{u_{r}}{r^{2}} \frac{\partial\left(r^{2} B_{r}\right)}{\partial r}+\vec{B}_{h} \cdot \nabla_{h} u_{r}
$$

The term on the left-hand side of (10) is the frozenflux SV, i.e., the change in the radial field due to the flow. The first and third terms on the right-hand side of (10) represent the tangential and radial magnetic advection, respectively. The second and fourth terms on the right-hand side of (10) represent the tangential and radial magnetic field stretching, respectively.

We note that it is possible to rewrite the radial advection term using $\nabla \cdot \vec{B}=0$ as $u_{r} \nabla_{h} \cdot \vec{B}_{h}$. However, in fluid dynamics, advection is affiliated with the flow interacting with a gradient of a tracer (in this case the magnetic field). In our radial advection term that is precisely the case, whereas expressing this term using a divergence is less intuitive.

Figure 1 illustrates the four SV mechanisms at depth. Tangential advection $A d_{h}$ occurs due to the displacement of the radial magnetic field on the spherical surface when a tangential flow has a component perpendicular to the $B_{r}$-isolines (Fig. 1a). This process produces bipolar features in the SV (Amit 2014; Livermore et al. 2017). Tangential stretching $S t_{h}$ occurs due to the interaction of $B_{r}$ with downwelling or upwelling structures (Fig. 1b). Downwelling intensifies the radial magnetic field (Christensen et al. 1998), resulting in same-sign polarity features in the SV (Amit 2014; Peña et al. 2016). Similar to $A d_{h}$, radial advection $A d_{r}$ occurs due to the radial displacement of $B_{r}$ by the radial flow (Fig. 1c). Radial stretching $S t_{r}$ occurs due 


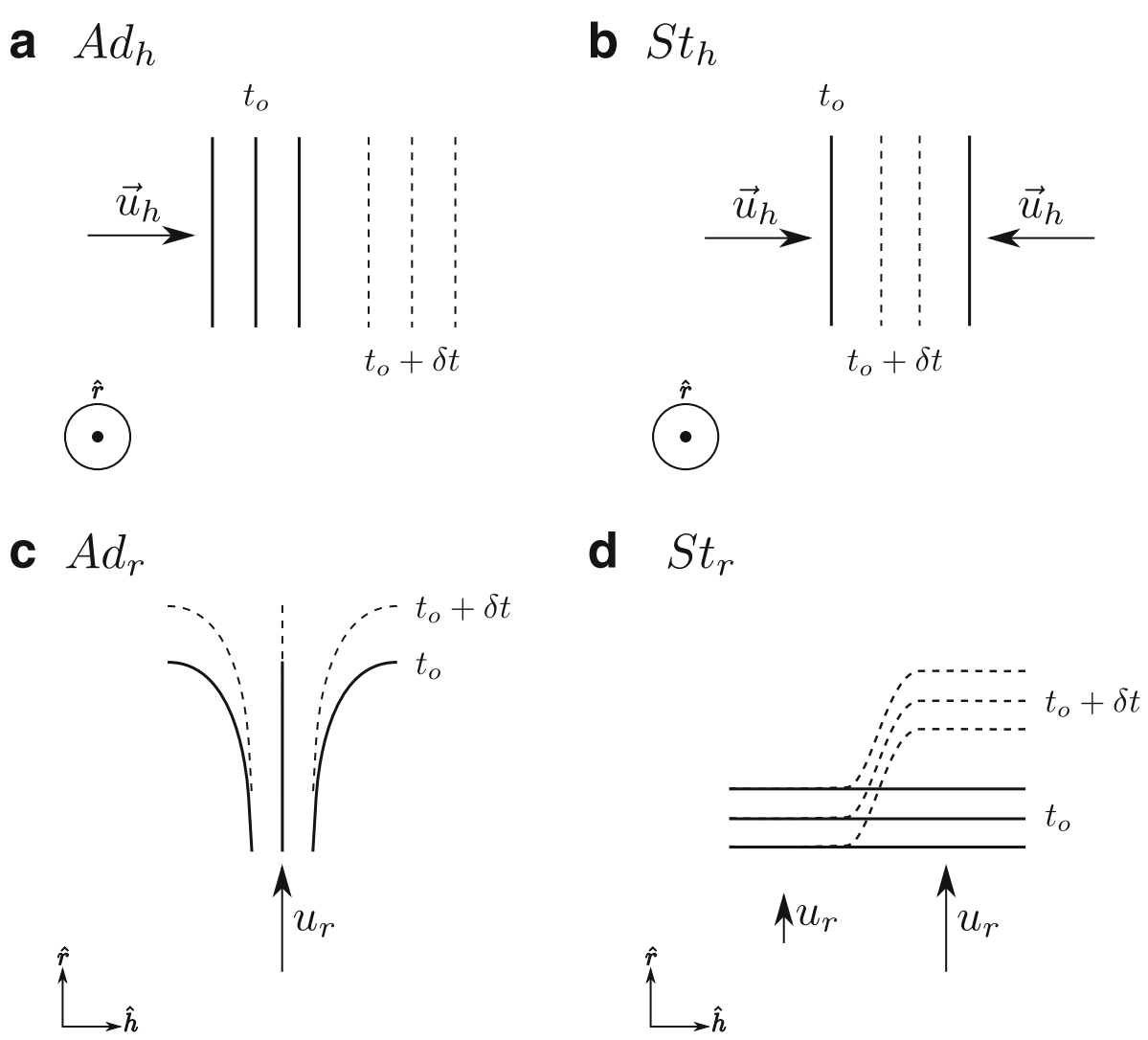

Fig. 1 Schematic illustrations of the radial frozen-flux SV mechanisms. a Tangential advection. b Tangential stretching. c Radial advection. d Radial stretching. The solid lines represent the initial configuration at time $t_{0}$, and the dashed lines represent an evolved configuration due to the fluid flow action after some time $\delta t$. The lines represent either radial field contours ( $\mathbf{a}$ and $\mathbf{b}$ ) or magnetic field lines ( $\mathbf{c}$ and $\mathbf{d}$ ). Spatial orientations are given at the bottom left of each subplot

to the interaction of tangential magnetic field lines with tangentially varying radial flow (Fig. 1d). In this dynamo mechanism, the laterally varying radial flow shears the toroidal field lines to generate a poloidal magnetic field (Olson et al. 1999).

At the CMB $\left(r=r_{o}\right.$, where $r_{o}$ is the outer core radius), the radial velocity vanishes $\left(u_{r}=0\right)$. Then, the radial advection and radial stretching terms (third and fourth terms on the right-hand side of (10), respectively) also vanish, and the radial component of the magnetic induction equation becomes (e.g. Bloxham and Jackson 1991; Holme 2015)

$$
{\frac{\partial B_{r} f f}{\partial t}}^{f f}=-\vec{u}_{h} \cdot \nabla_{h} B_{r}-B_{r} \nabla_{h} \cdot \vec{u}_{h}
$$

\section{Tangent cylinder at depth}

The presence of the inner core affects the geometry of the fluid flow in the outer core. The tangent cylindera hypothetical cylinder coaxial with Earth's rotation axis and tangential to the inner core at the equatorial plane (Fig. 2)-separates the outer core into two regions in which the fluid flow and the resulting magnetic field are expected to be quite different (e.g. Aurnou et al. 2003). In particular, the tangent cylinder rim acts as a flow barrier, resulting in surface convergence and intense magnetic flux patches in its intersection with the CMB (e.g. Olson et al. 1999).

Because the inner to outer radius ratio is $r_{i} / r_{o}=$ 0.35 (Dziewonski and Anderson 1981), the intersection of the tangent cylinder with the CMB occurs at colatitude $\theta_{o}^{c} \sim 20.5^{\circ}\left(\sin \theta_{o}^{c}=0.35 / 1\right.$, see Fig. 2$)$. At middepth $\left(r_{1 / 2} / r_{o}=0.675\right.$, where $r_{1 / 2}$ is the radius at middepth), the intersection occurs at colatitude $\theta_{1 / 2}^{c} \sim 31.2^{\circ}$ $\left(\sin \theta_{1 / 2}^{c}=0.35 / 0.675\right)$. The difference between these two angles implies that robust features on the CMB (e.g., high-latitude intense flux patches) may have their roots at mid-depth at lower latitudes if these features are related to the tangent cylinder rim effect.

\section{Image distortion}

When considering the same longitudinal and latitudinal extents for both the outer boundary and mid-depth patches, a root (at mid-depth) of an intense flux patch (at the CMB) would exhibit an apparent distortion due to 


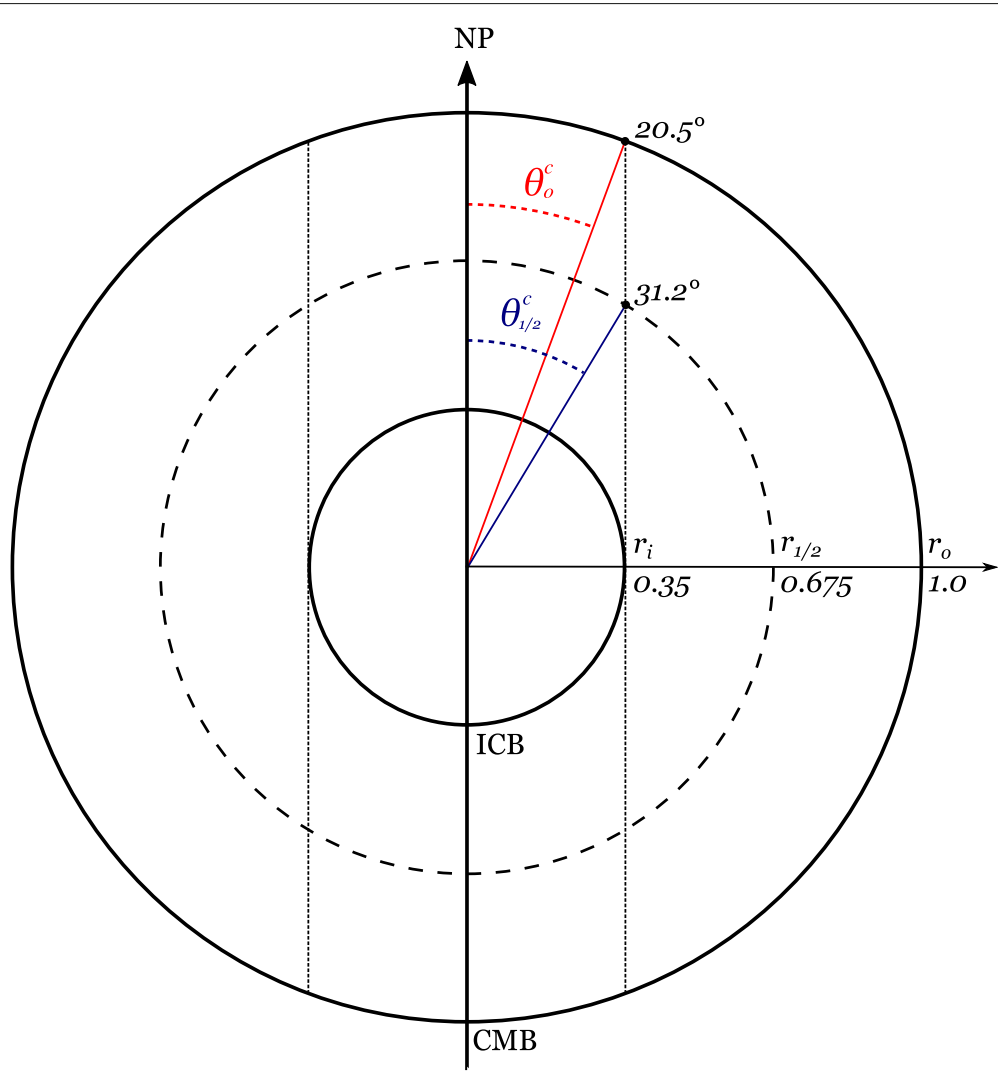

Fig. 2 Schematic illustration of the inner core tangent cylinder and its intersection with the CMB (red) and with the spherical surface at mid-shell (blue)

the difference between the corresponding CMB and middepth areas of equal angular extents. This difference is associated with the change in radial level from the $\mathrm{CMB}$ to mid-depth as well as a possible change in latitude. The root occupies a larger portion of the angular surface than a CMB structure of the same size. This radial distortion ratio is given by

$$
\left(\frac{r_{o}}{r_{1 / 2}}\right)^{2}=\left(\frac{1}{0.675}\right)^{2}
$$

Such a distortion, which we will refer to as plume-like, should conserve the spatial angle, i.e., $\delta \vartheta=\delta \varphi=0$, where $\delta \vartheta$ and $\delta \varphi$ are the latitudinal and longitudinal shifts, respectively, between the root and the outer boundary patches. In contrast, if the roots reside at lower latitudes than the $\mathrm{CMB}$ structures, e.g., due to a tangent cylinder rim effect, the roots appear relatively smaller. The purely latitudinal distortion from one $\mathrm{CMB}$ patch centered at the intersection of the tangent cylinder and the $\mathrm{CMB}$ to another $\mathrm{CMB}$ patch centered at the intersection of the tangent cylinder and the mid-depth spherical surface is given by $\frac{\sin \theta_{c}^{c}}{\sin \theta_{1 / 2}^{c}}=0.675$.
The ratio between the areas of a structure at the $\mathrm{CMB}$ $S_{o}$ and its root at mid-depth $S_{1 / 2}$ with both structures centered at the tangent cylinder, which we will refer to as the tangent cylinder rim effect, is

$$
\frac{S_{o}}{S_{1 / 2}}=\left(\frac{r_{o}}{r_{1 / 2}}\right)^{2} \cdot \frac{\sin \theta_{o}^{c}}{\sin \theta_{1 / 2}^{c}}=\frac{1}{0.675}
$$

For a general latitudinal distortion combined with the radial distortion, we calculated the correlation between a pair of CMB and mid-depth field structures for a range of $S_{o} / S_{1 / 2}$ (Fig. 3). In Fig. 3a, we show two examples of these distortions: a root with a larger area and a root with a smaller area representing a purely radial and a purely latitudinal distortion, respectively. Note that the latter is hypothetical since radial distortion is always present. In practice, for a latitudinal distortion that ranges from zero to a tangent cylinder rim effect (as we will later show is the case), the combined radial and latitudinal distortion is represented by the gap between the green and red vertical lines in Fig. 3b. According to these results, the correlation between a flux patch at the CMB and its root at middepth is high, in the range $0.77-0.93$. This indicates that it is possible to identify a root despite the geometrical 


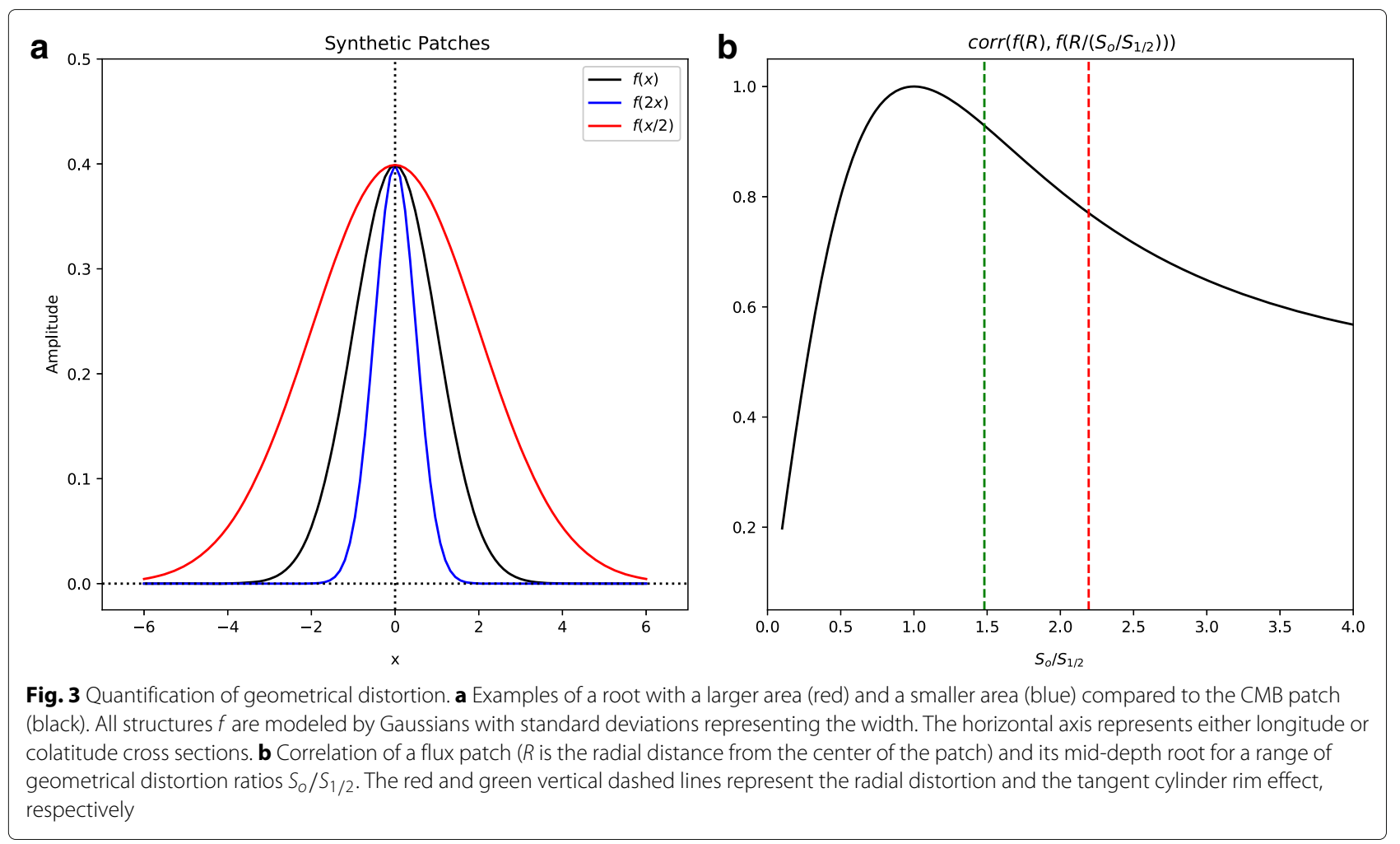

distortions, as long as the patch is not too distorted by the dynamics in the shell.

\section{Numerical dynamos}

We use the same dynamo models as in Peña et al. (2016). Here, we briefly recall the description of these models (for further details, see Peña et al. 2016). Numerical dynamos are solutions to the magnetohydrodynamics equations: Navier-Stokes, magnetic induction, conservation of energy, conservation of mass (continuity for an incompressible fluid), and no magnetic monopoles. In non-dimensional form, these equations can be written (e.g. Olson et al. 1999) as follows:

$$
\begin{gathered}
E\left(\frac{\partial \vec{u}}{\partial t}+\vec{u} \cdot \nabla \vec{u}-\nabla^{2} \vec{u}\right)+2 \hat{z} \times \vec{u}+\nabla P \\
=R a \frac{\vec{r}}{r_{o}} T+\frac{1}{P m}(\nabla \times \vec{B}) \times \vec{B} \\
\frac{\partial \vec{B}}{\partial t}=\nabla \times(\vec{u} \times \vec{B})+\frac{1}{P m} \nabla^{2} \vec{B} \\
\frac{\partial T}{\partial t}+\vec{u} \cdot \nabla T=\frac{1}{P r} \nabla^{2} T+\epsilon
\end{gathered}
$$

$$
\nabla \cdot \vec{u}=0
$$

$$
\nabla \cdot \vec{B}=0
$$

where $\vec{u}$ is the fluid velocity, $\vec{B}$ is the magnetic field, $T$ is the temperature (or more generally co-density), $t$ is the time, $\hat{z}$ is a unit vector in the direction of the rotation axis, $P$ is the pressure, $\vec{r}$ is the position vector, and $\epsilon$ is a heat (or buoyancy) source or sink.

Four non-dimensional parameters in (14)-(16) control the dynamo action. The heat flux Rayleigh number (Olson and Christensen 2002) represents the strength of the buoyancy force driving the convection relative to retarding forces

Table 1 Dynamo models control parameters: Rayleigh $R a$, Ekman E, and magnetic Prandtl Pm

\begin{tabular}{llllll}
\hline Model & $R a$ & $E$ & $P m$ & $R m$ & $\bar{\delta} \tau$ \\
\hline 1 & $2 \times 10^{5}$ & $1 \times 10^{-3}$ & 5 & 137 & 14.75 \\
2 & $2 \times 10^{5}$ & $1 \times 10^{-3}$ & 10 & 255 & 11.9 \\
3 & $4 \times 10^{5}$ & $1 \times 10^{-3}$ & 5 & 219 & 33.07 \\
4 & $5 \times 10^{5}$ & $3 \times 10^{-4}$ & 3 & 82 & 22.51 \\
5 & $1 \times 10^{6}$ & $3 \times 10^{-4}$ & 3 & 125 & 14.60 \\
6 & $3 \times 10^{6}$ & $3 \times 10^{-4}$ & 3 & 234 & 78.98 \\
7 & $1 \times 10^{7}$ & $1 \times 10^{-4}$ & 1.3 & 126 & 9.42 \\
8 & $1 \times 10^{7}$ & $1 \times 10^{-4}$ & 2 & 218 & 5.50 \\
9 & $3 \times 10^{7}$ & $1 \times 10^{-4}$ & 2 & 446 & 16.66 \\
\hline
\end{tabular}

For all models, we set the Prandtl number to $\operatorname{Pr}=1 . \bar{\delta} \bar{\tau}$ denotes the average time difference between successive snapshots in units of magnetic advection time 

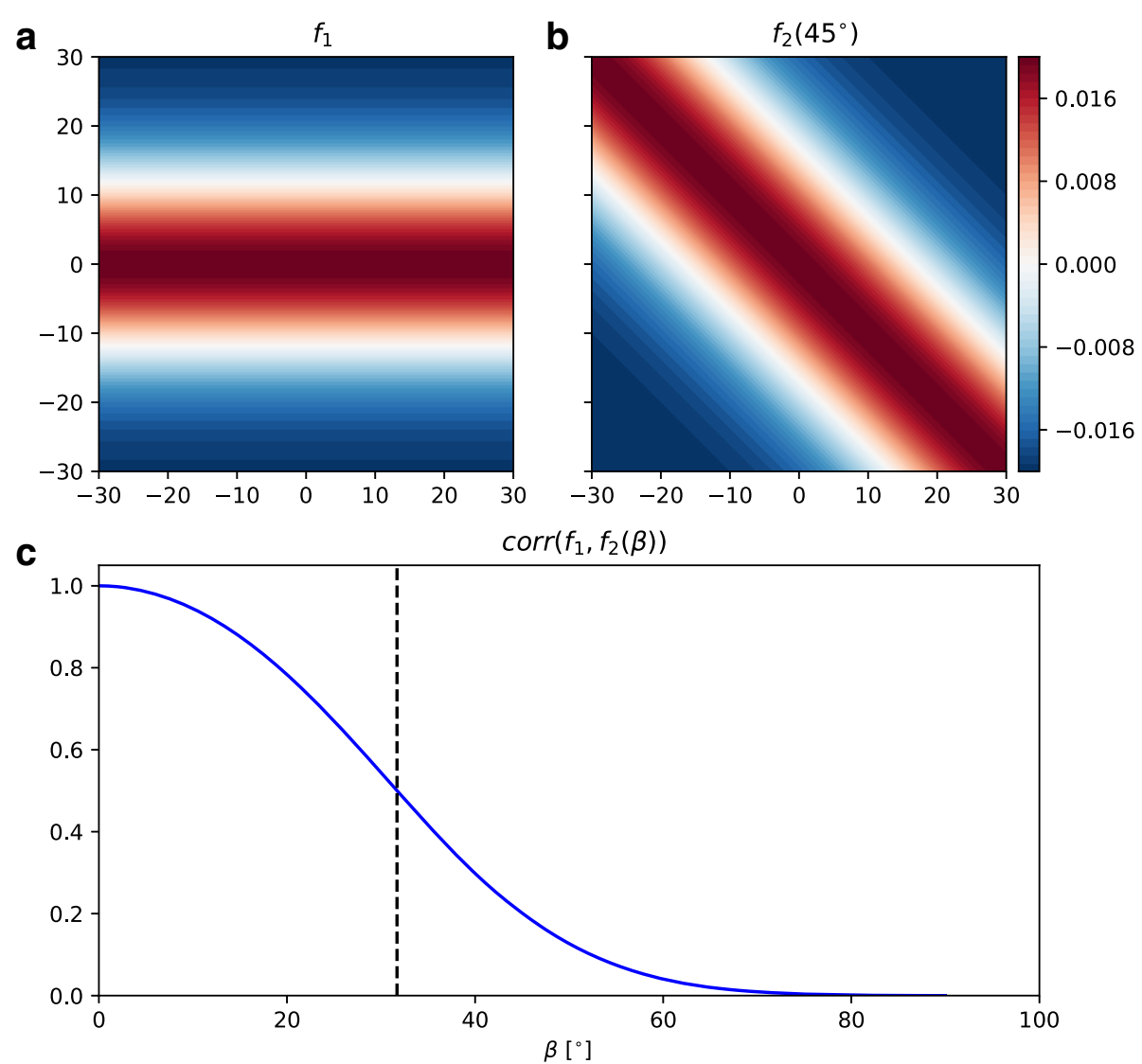

Fig. 4 Same polarity image distortion. a An example of a synthetic intense flux patch at the CMB. $\mathbf{b}$ Its root at mid-depth rotated by $45^{\circ}$. The patch is modeled by a Gaussian with standard deviation equals to 1. c Correlation between the intense flux patch in $\mathbf{a}$ and its root rotated by $\alpha$. The black dashed line shows the maximum acceptable rotation $\beta \approx 31.7^{\circ}$ for correlations higher than 0.5

$$
R a=\frac{\alpha g_{o} q_{o} D^{4}}{k \kappa v}
$$

where $\alpha$ is the thermal expansivity, $g_{o}$ is the gravitational acceleration on the outer boundary at radius $r_{o}, q_{o}$ is the mean heat flux across the outer boundary, $D$ is the shell thickness, $k$ is the thermal conductivity, $\kappa$ is the thermal diffusivity, and $v$ is the kinematic viscosity. The Ekman number represents the ratio of viscous to Coriolis forces

$$
E=\frac{v}{\Omega D^{2}}
$$

Table 2 Global statistics at mid-depth: dynamo models time average and standard deviation values

\begin{tabular}{lllllll}
\hline Model & $A d_{r} / A d_{h}$ & $S t_{r} / S t_{h}$ & $S t_{h} / A d_{h}$ & $S t / A d$ & $\mathcal{P} / \mathcal{T}$ & $\left\|.4 u_{r}\right\| /\left\|\vec{u}_{h}\right\|$ \\
\hline 1 & $0.63 \pm 0.05$ & $1.33 \pm 0.22$ & $0.44 \pm 0.05$ & $0.78 \pm 0.16$ & $0.37 \pm 0.04$ & $0.69 \pm 0.06$ \\
2 & $0.60 \pm 0.10$ & $1.26 \pm 0.20$ & $0.41 \pm 0.07$ & $0.66 \pm 0.10$ & $0.38 \pm 0.04$ & $0.71 \pm 0.04$ \\
3 & $0.63 \pm 0.08$ & $1.29 \pm 0.18$ & $0.38 \pm 0.06$ & $0.65 \pm 0.12$ & $0.43 \pm 0.03$ & $0.76 \pm 0.10$ \\
4 & $0.50 \pm 0.07$ & $1.76 \pm 0.22$ & $0.43 \pm 0.08$ & $0.90 \pm 0.12$ & $0.32 \pm 0.04$ & $0.60 \pm 0.06$ \\
5 & $0.52 \pm 0.07$ & $1.45 \pm 0.17$ & $0.48 \pm 0.07$ & $0.85 \pm 0.09$ & $0.38 \pm 0.03$ & $0.65 \pm 0.06$ \\
6 & $0.64 \pm 0.07$ & $1.25 \pm 0.15$ & $0.50 \pm 0.05$ & $0.81 \pm 0.10$ & $0.42 \pm 0.05$ & $0.75 \pm 0.05$ \\
7 & $0.57 \pm 0.08$ & $1.27 \pm 0.11$ & $0.64 \pm 0.11$ & $1.00 \pm 0.14$ & $0.38 \pm 0.03$ & $0.71 \pm 0.06$ \\
8 & $0.59 \pm 0.05$ & $1.29 \pm 0.13$ & $0.56 \pm 0.04$ & $0.88 \pm 0.13$ & $0.42 \pm 0.05$ & $0.74 \pm 0.07$ \\
9 & $0.65 \pm 0.04$ & $1.14 \pm 0.07$ & $0.52 \pm 0.05$ & $0.75 \pm 0.06$ & $0.44 \pm 0.04$ & $0.79 \pm 0.05$ \\
\hline
\end{tabular}

$S t_{r}, S t_{h}$, and $S t$ are the radial, tangential, and total stretching $r m s$, respectively; $A d_{r}, A d_{h}$, and $A d$ are the radial, tangential, and total advection $r m s$, respectively; $\mathcal{P} / \mathcal{T}$ is the poloidal/toroidal flow rms ratio, and $\left\|u_{r}\right\| /\left\|\vec{u}_{h}\right\|$ is the radial/tangential flow rms ratio 
where $\Omega$ is the rotation rate. The Prandtl number is the ratio of kinematic viscosity to thermal diffusivity

$$
\operatorname{Pr}=\frac{v}{\kappa}
$$

and the magnetic Prandtl number is the ratio of kinematic viscosity to magnetic diffusivity

$$
P m=\frac{v}{\lambda}
$$

The most important output parameter is the magnetic Reynolds number $R m$, which represents the scaled ratio between magnetic advection and diffusion and is given by

$$
R m=\frac{U D}{\lambda}
$$

where $U$ is a typical velocity scale.

We used the numerical implementation MagIC (Wicht 2002). Due to computational limitations, dynamo simulations use control parameters very far from Earth-like conditions, therefore relating the results to the real core conditions is challenging. Our chosen control parameters (Table 1 ) are moderate compared to what modern computers are capable of. The reason is that smaller $E$

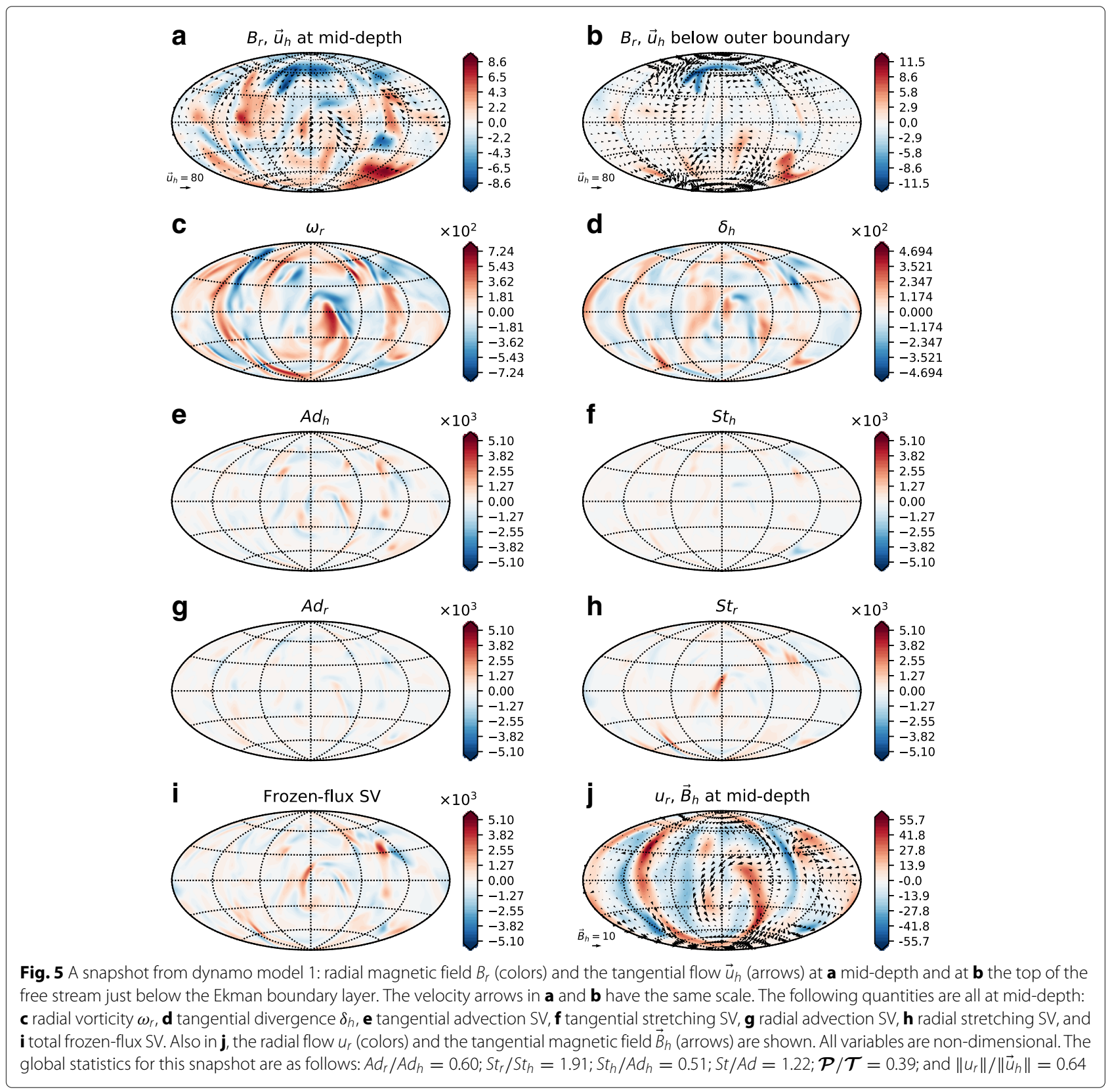


values produce such small-scale structures that the local relations between the field and the flow would become difficult to interpret. We focus on dynamos in the nonreversing dipole-dominated regime (e.g. Christensen and Aubert 2006; Kutzner and Christensen 2002).

The shell geometry is identical to Earth's core, with an inner to outer boundary radius ratio of $r_{i} / r_{o}=0.35$. The inner and outer boundaries of the shell are set to be insulating and rigid. To simulate generic thermo-chemical convection (e.g. Aubert et al. 2008a), on the inner core boundary, a fixed co-density is set; on the outer boundary, a fixed heat flux is prescribed; and the source/sink term in (15) is set to $\epsilon=0$. The number of radial grid points $N_{r}$ is chosen to accommodate at least five grid points across the Ekman boundary layer. In our models, $N_{r}$ varies from 49 for the larger $E=1 \times 10^{-3}$ cases to 61 for the smaller $E=1 \times 10^{-4}$ cases. Horizontal resolution is also increased with a decreasing Ekman number, from a maximum degree and order $\ell_{\max }=64$ for the $E=1 \times 10^{-3}$ cases to $\ell_{\max }=96$ for the $E=1 \times 10^{-4}$ cases.

\section{Criterion for root identification}

In order to identify deep roots of intense flux patches on the outer boundary, we used the identified structures at the outer boundary which were studied by Peña et al. (2016). These outer boundary $B_{r}$ structures were compared with $B_{r}$ structures at mid-depth using an auto-correlation function for the same longitudinal and latitudinal extent. Our algorithm calculates the Pearson correlation (e.g. Press et al. 1992) between local $B_{r}$ on the outer boundary and local $B_{r}$ at depth

$$
\frac{\sum_{i}\left(B_{r, o}^{i}-\overline{B_{r, o}^{i}}\right)\left(B_{r, 1 / 2}^{i}-\overline{B_{r, 1 / 2}^{i}}\right)}{\sqrt{\sum_{i}\left(B_{r, o}^{i}-\overline{B_{r, o}^{i}}\right)^{2}} \sqrt{\sum_{i}\left(B_{r, 1 / 2}^{i}-\overline{B_{r, 1 / 2}^{i}}\right)^{2}}}
$$

In (24), $i$ denotes a set of points $\left(\theta_{i}, \varphi_{i}\right)$ defined at both $r_{0}$ and $r_{1 / 2}$. We consider a displacement of the center of the latter of up to $15^{\circ}$ in colatitude and longitude while conserving the longitudinal and latitudinal extents. The best correlation determines the location of the patch at depth. Structures with correlations higher than a critical threshold are defined as roots.

As shown in Fig. 3, the deterioration of the correlation due to geometrical distortion is expected to be minor. The opposite problem of biased high correlations might occur merely because same-polarity $\mathrm{CMB}$ and deep structures are considered. For example, deep structures rotated with respect to a CMB patch are nevertheless positively correlated. However, a large angle representing a false root would result in a reduced correlation coefficient between the two radial field structures (see Fig. 4a, b for an example with an angle of $45^{\circ}$ ). For this study, we accept roots with a correlation higher than an arbitrary threshold value of 0.5 (black dashed line in Fig. 4c) that corresponds to an angle of $\sim 31.7^{\circ}$. With this selection process, we avoid falsely attributing roots to positive correlations that originate from merely same-sign structures.

\section{Statistics}

As in Peña et al. (2016), we calculated global and local rms ratios $(\|X\| /\|Y\|)$ between pairs of quantities $X$ and $Y$. The rms $\|X\|$ is obtained by integration of $X$ over the spherical surface at mid-shell. In addition to the statistical measures calculated by Peña et al. (2016), we also quantified SV terms that appear at depth but vanish on approach to the boundary. These include the radial stretching to tangential stretching rms ratio $S t_{r} / S t_{h}$ and radial advection to tangential advection rms ratio $A d_{r} / A d_{h}$. We also quantified the rms ratio of radial to tangential velocity $\left\|u_{r}\right\| /\left\|\vec{u}_{h}\right\|$.

We used the same local classification by polarity and by latitude as in Peña et al. (2016). Polarity is defined as normal and reversed with respect to the sign of the axial dipole. High latitudes are arbitrarily defined by patches that are centered at latitudes higher than $45^{\circ}$. Classified this way, four types of patches are possible: normal polarity at high latitudes $(\mathrm{HN})$, normal polarity at low latitudes (LN), reversed polarity at high latitudes (HR), and reversed polarity at low latitudes (LR). Note that we classified the deep structures according to the latitudes of the correlated outer boundary structures even if the roots are found on the other side of latitude $45^{\circ}$.

To examine a possible tangent cylinder rim effect, we calculated the dislocation of pairs of outer boundary and mid-depth patches. We defined $\delta \varphi$ and $\delta \vartheta$ as the longitudinal and latitudinal dislocation of patches

Table 3 Identified roots: detection fraction in percentage of roots at mid-depth of the intense magnetic flux patches at the top of the shell reported by Peña et al. (2016) in each dynamo model

\begin{tabular}{lllll}
\hline Model & Total & HN & LN & LR \\
\hline 1 & 82.4 & 77.5 & 100 & 80 \\
2 & 65.3 & 70.8 & 40 & 57.9 \\
3 & 60.6 & 60.7 & - & 60 \\
4 & 44.4 & 55.2 & 0 & 0 \\
5 & 71.4 & 80 & 0 & 0 \\
6 & 62.5 & 64.5 & 66.7 & 50 \\
7 & 85.2 & 95.2 & 66.7 & 33.3 \\
8 & 38.7 & 37.9 & - & 50 \\
9 & 20 & 15.4 & 25 & 40
\end{tabular}

The detected roots are classified on the basis of the latitudes of the magnetic flux patches on the outer boundary: high-latitude normal intense flux patches (HN), low-latitude normal intense flux patches (LN), and low-latitude reversed flux patches (LR) 
from the outer boundary to mid-depth, respectively. Positive/negative $\delta \vartheta$ corresponds to lower/higher latitudes of mid-depth patches, respectively.

Finally, we examined the dependence of the statistical quantities on the non-dimensional control parameters of the dynamo models. Each quantity may be expressed as a generic power law (as in Peña et al. 2016):

$$
f=C \cdot E^{a} \cdot R a^{b} \cdot P m^{c}
$$

where $f$ is the statistical quantity and $C, a, b$, and $c$ are fitting coefficients. The relative misfit $\sigma_{r}$ of the power law is given by

$$
\sigma_{r}=\sqrt{\sum_{i=1}^{n}\left(f_{i}^{\mathrm{dyn}}-f_{i}\right)^{2} / \sum_{i=1}^{n}\left(f_{i}^{\mathrm{dyn}}\right)^{2}}
$$

where $f^{\text {dyn }}$ is the statistical quantity obtained from the dynamo models and $n$ is the number of dynamo

\begin{tabular}{|c|c|c|c|c|c|c|}
\hline Model & PT & $A d_{h}$ & $S t_{h}$ & $A d_{r} 666666$ & $S t_{r}$ & All \\
\hline & $\mathrm{HN}$ & $3.37 \pm 5.06$ & $7.50 \pm 0.00$ & $5.62 \pm 0.00$ & $3.44 \pm 6.71$ & $3.58 \pm 5.31$ \\
\hline \multirow[t]{3}{*}{1} & LN & $-15.00 \pm 0.00$ & - & - & - & $-15.00 \pm 0.00$ \\
\hline & $L R$ & $-3.00 \pm 6.67$ & $5.62 \pm 0.00$ & $-1.88 \pm 1.88$ & - & $-1.64 \pm 6.04$ \\
\hline & $\mathrm{HN}$ & $4.31 \pm 4.32$ & $-1.88 \pm 0.00$ & $5.62 \pm 0.00$ & $5.63 \pm 3.75$ & $4.25 \pm 4.75$ \\
\hline \multirow[t]{3}{*}{2} & $L N$ & $-7.50 \pm 1.88$ & - & - & - & $-7.50 \pm 1.88$ \\
\hline & $L R$ & $-0.19 \pm 7.48$ & $-15.00 \pm 0.00$ & - & - & $-1.53 \pm 8.30$ \\
\hline & $\mathrm{HN}$ & $2.46 \pm 6.91$ & $5.62 \pm 0.00$ & - & - & $2.65 \pm 6.75$ \\
\hline \multirow[t]{3}{*}{3} & LN & - & - & - & - & - \\
\hline & LR & $3.75 \pm 0.00$ & $5.62 \pm 7.50$ & - & - & $5.00 \pm 6.19$ \\
\hline & $\mathrm{HN}$ & $11.25 \pm 5.81$ & - & $10.00 \pm 3.85$ & $5.86 \pm 4.44$ & $8.32 \pm 5.42$ \\
\hline \multirow[t]{3}{*}{4} & $L N$ & - & - & - & - & - \\
\hline & $L R$ & - & - & - & - & - \\
\hline & $\mathrm{HN}$ & $8.04 \pm 2.18$ & - & $7.50 \pm 1.33$ & $10.21 \pm 2.67$ & $8.91 \pm 2.58$ \\
\hline \multirow[t]{3}{*}{5} & $L N$ & - & - & - & - & - \\
\hline & $L R$ & - & - & - & - & - \\
\hline & $\mathrm{HN}$ & $5.25 \pm 5.82$ & $11.25 \pm 0.00$ & $11.25 \pm 0.00$ & $-1.87 \pm 8.53$ & $4.78 \pm 6.87$ \\
\hline \multirow[t]{3}{*}{6} & LN & $13.13 \pm 1.88$ & - & - & - & $13.13 \pm 1.88$ \\
\hline & LR & $-5.00 \pm 5.79$ & - & - & - & $-5.00 \pm 5.79$ \\
\hline & $\mathrm{HN}$ & $3.75 \pm 0.00$ & - & $8.44 \pm 0.94$ & $5.62 \pm 4.77$ & $5.81 \pm 4.51$ \\
\hline \multirow[t]{3}{*}{7} & LN & $0.94 \pm 0.94$ & - & - & - & $0.94 \pm 0.94$ \\
\hline & $L R$ & $1.88 \pm 0.00$ & - & - & - & $1.88 \pm 0.00$ \\
\hline & $\mathrm{HN}$ & $10.31 \pm 7.08$ & - & $0.00 \pm 0.00$ & $4.69 \pm 4.81$ & $6.31 \pm 6.46$ \\
\hline \multirow[t]{3}{*}{8} & LN & - & - & - & - & - \\
\hline & LR & $-7.50 \pm 0.00$ & - & - & - & $-7.50 \pm 0.00$ \\
\hline & $\mathrm{HN}$ & $-1.41 \pm 5.83$ & - & - & - & $-1.41 \pm 5.83$ \\
\hline \multirow[t]{3}{*}{9} & $\mathrm{LN}$ & $8.44 \pm 2.82$ & - & - & - & $8.44 \pm 2.82$ \\
\hline & LR & $-1.88 \pm 0.00$ & - & $5.62 \pm 0.00$ & - & $1.87 \pm 3.75$ \\
\hline & $\mathrm{HN}$ & $4.52 \pm 5.99$ & $5.62 \pm 4.78$ & $7.64 \pm 3.41$ & $5.66 \pm 5.70$ & $5.11 \pm 5.80$ \\
\hline \multirow[t]{2}{*}{$\bar{x}$} & LN & $1.67 \pm 9.62$ & - & - & - & $1.67 \pm 9.62$ \\
\hline & LR & $-1.62 \pm 6.82$ & $0.47 \pm 10.38$ & $0.62 \pm 3.85$ & - & $-1.10 \pm 7.25$ \\
\hline
\end{tabular}

Table 4 Latitudinal shift: mid-depth root latitudinal shift $\delta \vartheta$ with respect to the coordinates of the correlated outer boundary patch

Average and standard deviation values are given for each patch type (PT) and SV dominant mechanism. Positive values represent roots at lower latitudes, and negative values represent roots at higher latitudes. $\bar{x}$ denotes averages over all dynamo models, and "All" denotes averages over roots of all patch types 
models analyzed. Relative misfits larger than an arbitrary threshold value of 0.2 were considered inadequate, and in these cases, the fits were not interpreted.

The power law fits (25) obtained by the misfit minimization (26) were applied to time-average statistical quantities (Tables 2 and 5). The time dependence was expressed by the standard deviation $\sigma$. Note that $\sigma$ was not used to obtain the fits.

\section{Results}

Global stretching

Several statistical quantities were analyzed at mid-depth globally, i.e., over the spherical surface, for the nine
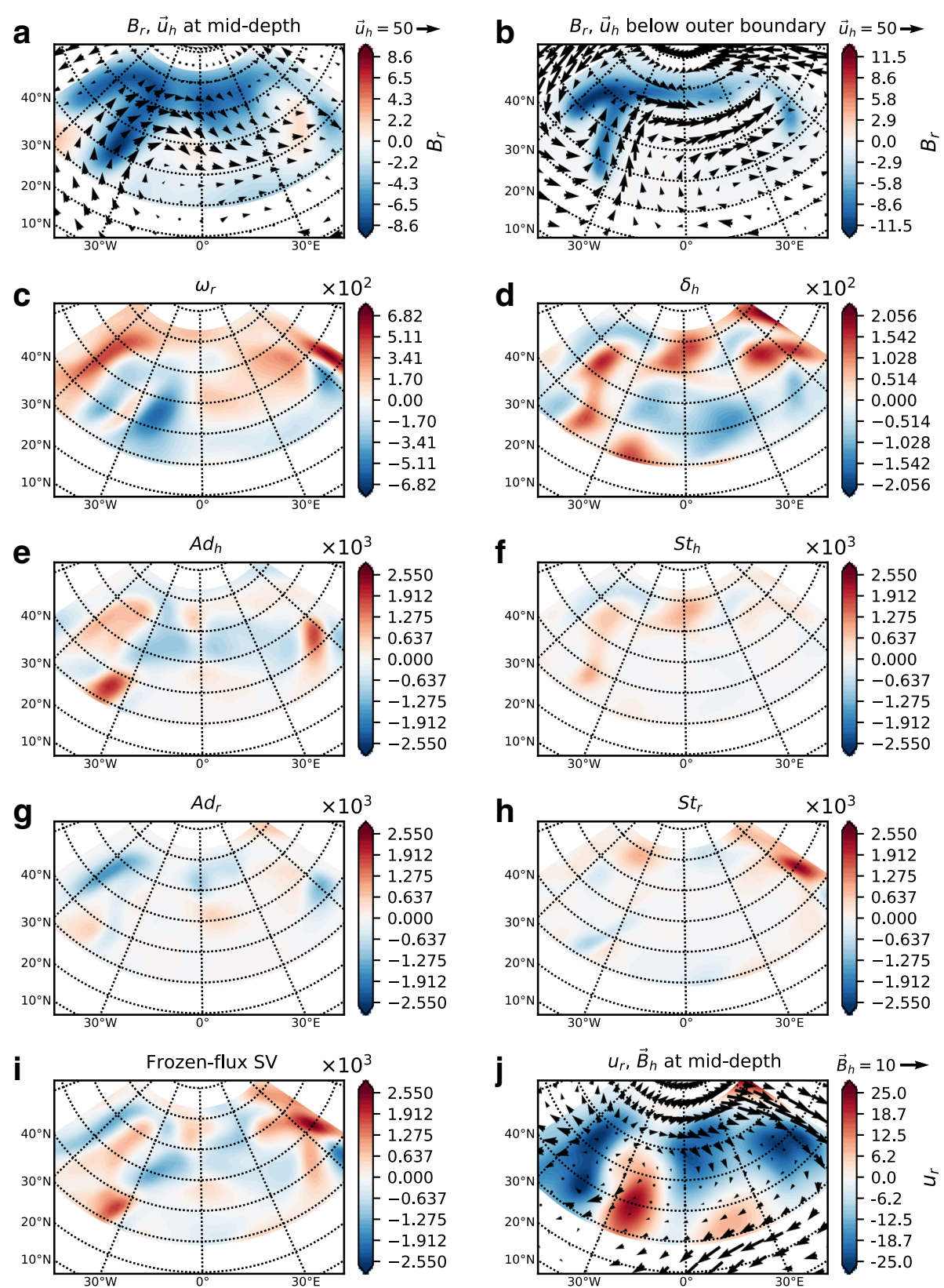

Fig. 6 Intense high-latitude normal polarity magnetic flux patch (HN) in dynamo model 1 (Fig. 5): radial magnetic field $B_{r}$ (colors) and the tangential flow $\vec{u}_{h}$ (arrows) at $\mathbf{a}$ mid-depth and at $\mathbf{b}$ the top of the free stream just below the Ekman boundary layer. The velocity arrows in $\mathbf{a}$ and $\mathbf{b}$ have the same scale. The following quantities are all at mid-depth: $\mathbf{c}$ radial vorticity $\omega_{r}, \mathbf{d}$ tangential divergence $\delta_{h}$, e tangential advection SV, $\mathbf{f}$ tangential stretching SV, $\mathbf{g}$ radial advection SV, $\mathbf{h}$ radial stretching SV, and $\mathbf{i}$ total frozen-flux SV. Also in $\mathbf{j}$, the radial flow $u_{r}$ (colors) and the tangential magnetic field $\vec{B}_{h}$ (arrows) are shown. All variables are non-dimensional. The mid-depth patch in $\mathbf{a}$ is shifted with respect to the outer boundary patch in $\mathbf{b}$ by $\delta \vartheta=1.88^{\circ}$ and $\delta \varphi=-1.88^{\circ}$ with an auto-correlation of 0.68 . The local statistics for this patch are as follows: $A d_{r} / A d_{h}=0.62 ; S t_{r} / S t_{h}=1.69$; $S t_{h} / A d_{h}=0.39 ; S t / A d=0.78 ; \mathcal{P} / \mathcal{T}=0.41 ;$ and $\left\|u_{r}\right\| /\left\|\vec{u}_{h}\right\|=0.62$ 
dynamo models described in Table 1. For each dynamo model, ten arbitrary snapshots well separated in time (see $\bar{\delta} \tau$ in Table 1) were analyzed.

We decomposed the tangential flow into toroidal and poloidal parts using $\vec{u}_{\text {tor }}=\nabla \times \mathcal{T} \hat{r}$ and $\vec{u}_{\text {pol }}=\nabla_{h} \mathcal{P}$, where $\mathcal{T}$ and $\mathcal{P}$ are the respective flow potentials. Note that in this decomposition, the radial poloidal flow is not considered. The ratio of rms flows $\left\|\vec{u}_{\text {pol }}\right\| /\left\|\vec{u}_{\text {tor }}\right\|$ is denoted by $\mathcal{P} / \mathcal{T}$.
The global statistics for each dynamo model are shown in Table 2. All cases exhibit a dominance of tangential advection $A d_{h}$ over radial advection $A d_{r}$ and a dominance of radial stretching $S t_{r}$ over tangential stretching $S t_{h}$. In addition, all models exhibit a total stretching/advection ratio of $S t / A d \sim 1.5-3$ times larger than the poloidal/toroidal flow ratio $\mathcal{P} / \mathcal{T}$ (Table 2).

Figure $5 \mathrm{a}, \mathrm{b}$ shows the radial field at mid-depth and at the outer boundary in a snapshot from dynamo model

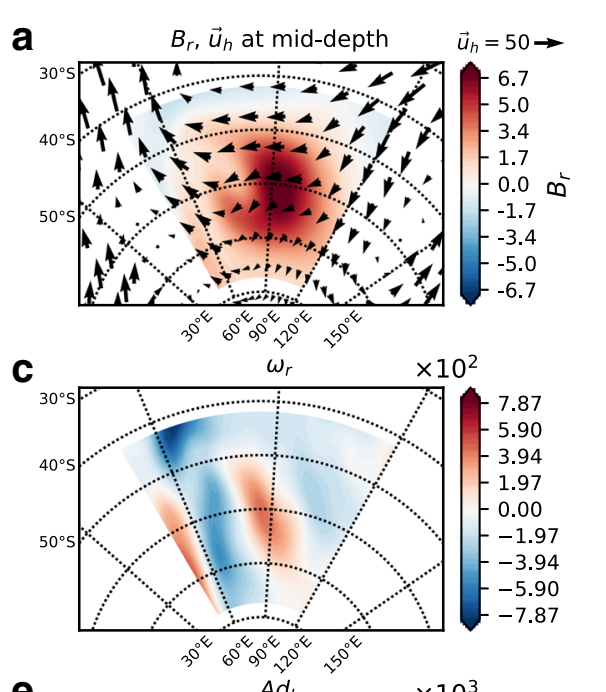

e

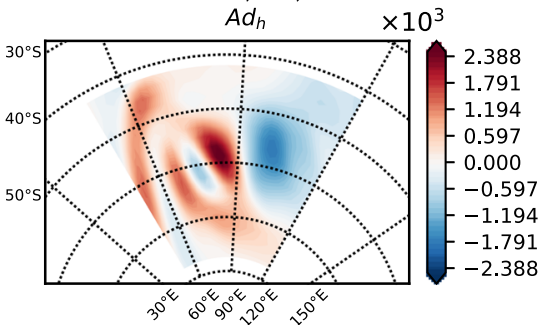

g

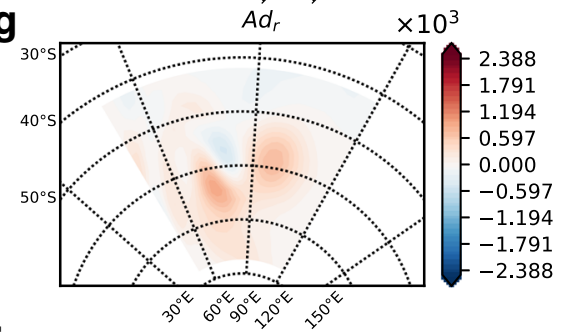

i

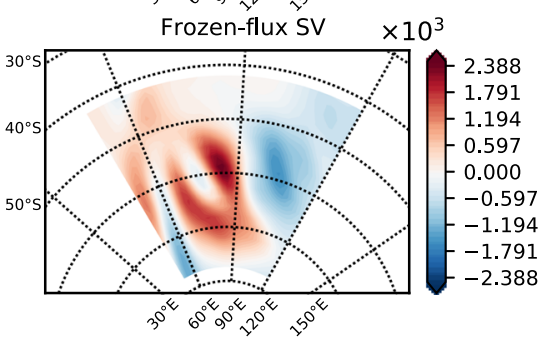

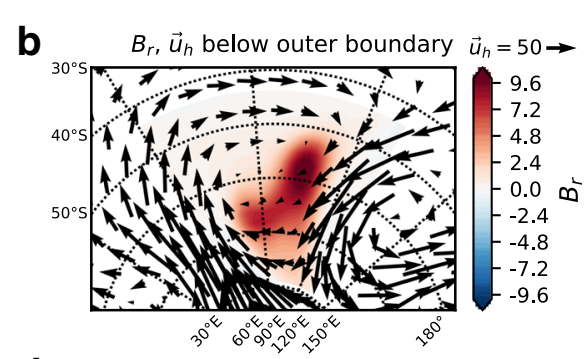

d

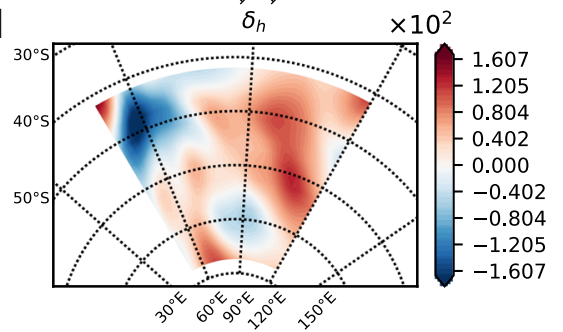

f

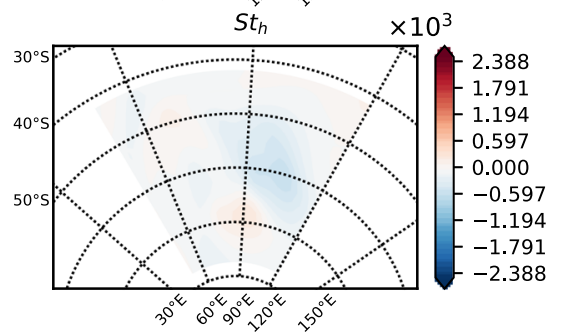

h

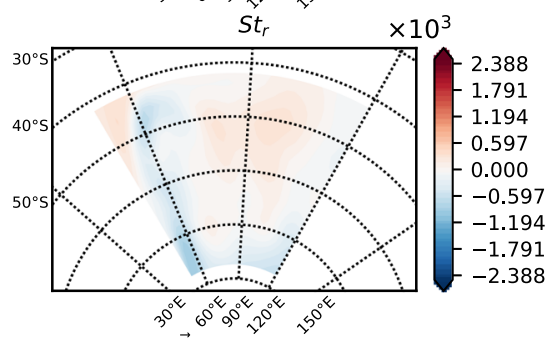

j

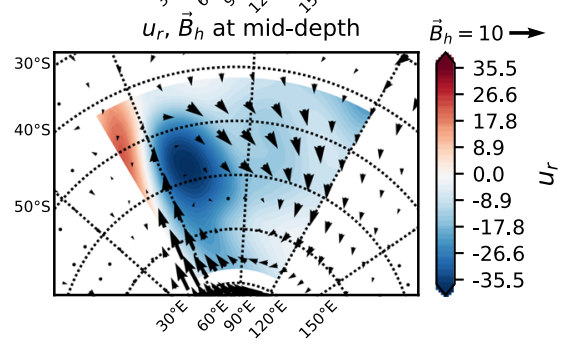

Fig. 7 As in Fig. 6 for another intense high-latitude normal polarity magnetic flux patch (HN). This patch presents a dominant $A d_{h}$ in dynamo model 3 . The mid-depth patch in $\mathbf{a}$ is shifted with respect to the outer boundary patch in $\mathbf{b}$ by $\delta \vartheta=1.88^{\circ}$ and $\delta \varphi=-9.38^{\circ}$ with an auto-correlation of 0.82 . The local statistics for this patch are as follows: $A d_{r} / A d_{h}=0.31 ; S t_{r} / S t_{h}=1.36 ; S t_{h} / A d_{h}=0.21 ; S t / A d=0.29 ; \mathcal{P} / \mathcal{T}=0.25 ;$ and $\left\|u_{r}\right\| /\left\|\vec{u}_{h}\right\|=0.50$ 
1. The field is much less concentrated with many more intense patches at mid-depth than at the outer boundary. Consequently, only a few intense patches previously detected at the outer boundary by Peña et al. (2016) could be identified at mid-depth. The radial vorticity $\omega_{r}=\hat{r}$. $\nabla \times \vec{u}$ (Fig. 5c) and the tangential divergence $\delta_{h}=\nabla_{h} \cdot \vec{u}_{h}$ (Fig. 5d) are weakly correlated, in contrast to the helical flow correlation (Amit and Olson 2004) observed at the top of the shell of numerical dynamos (Olson et al. 2002; Amit et al. 2007; Peña et al. 2016). The toroidal flow dominates over the poloidal, and the tangential flow dominates over the radial. Nevertheless, due to particular field-flow interactions, in this snapshot, the total stretching is larger than the total advection by a factor of 1.22 (see Fig. 5e-h).

\section{Kinematics of roots of intense magnetic flux patches}

Using the criteria described in the "Criterion for root identification" section, we identified and analyzed 213 mid-depth roots of outer boundary patches. Table 3 a

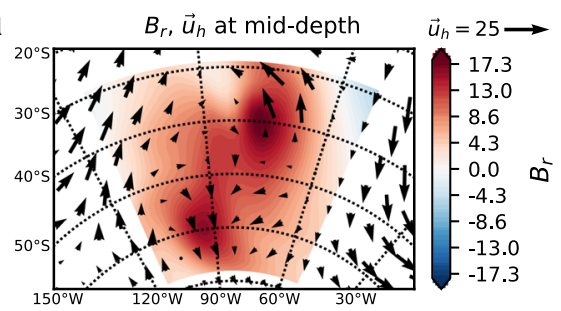

C

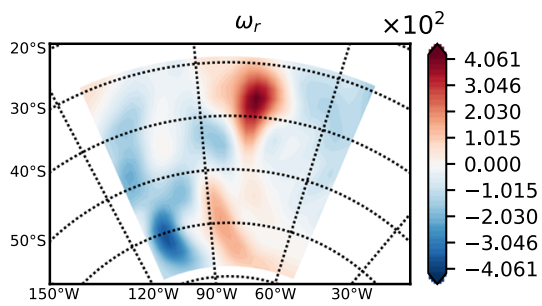

e

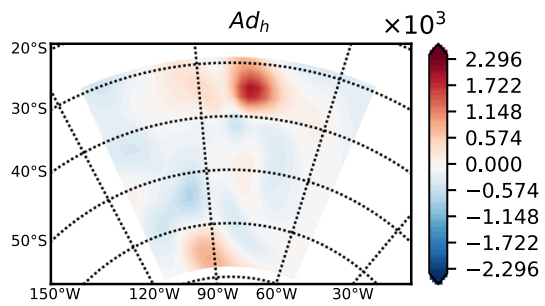

g

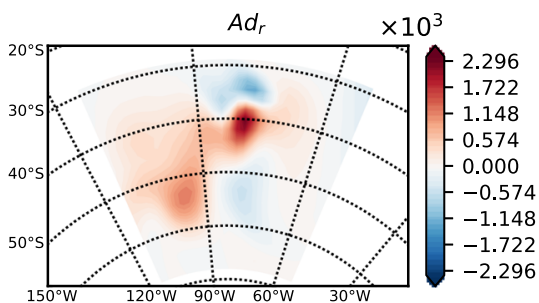

i

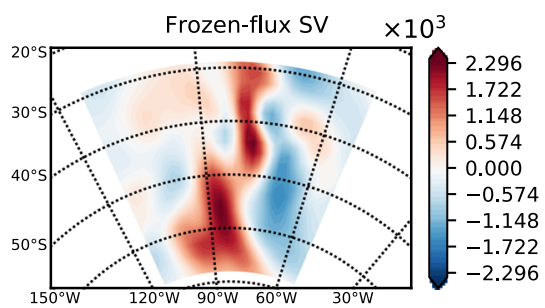

b

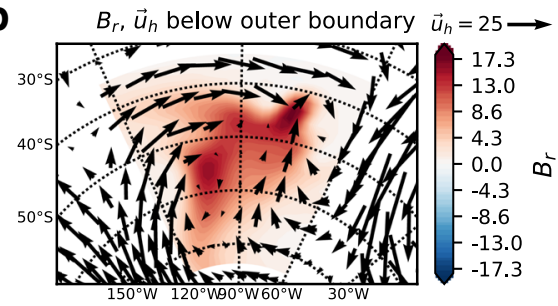

d

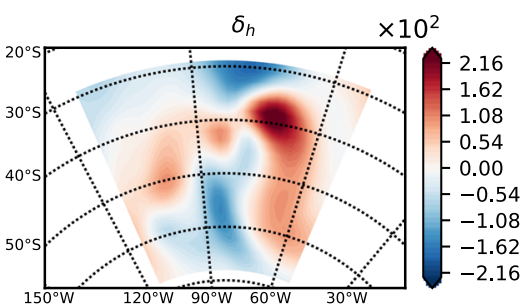

f

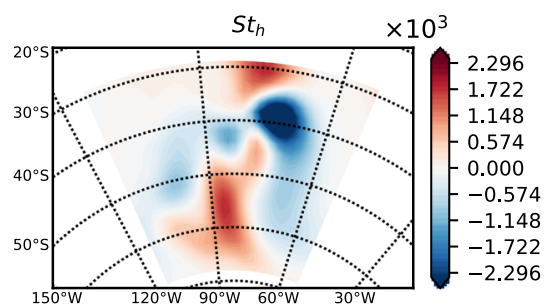

h

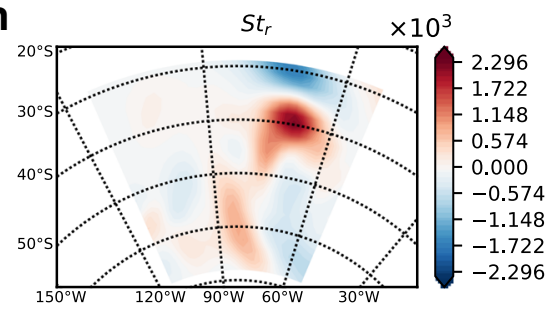

j

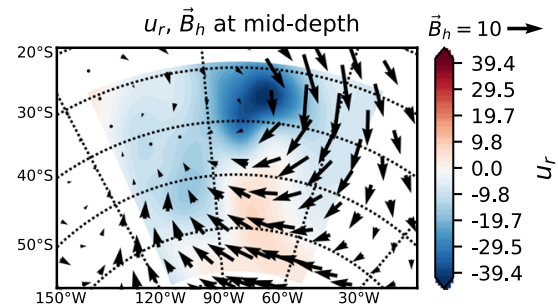

Fig. 8 As in Fig. 6 for another intense high-latitude normal polarity magnetic flux patch (HN). This patch presents a dominant Sth in dynamo model 2. The mid-depth patch in $\mathbf{a}$ is shifted with respect to the outer boundary patch in $\mathbf{b}$ by $\delta \vartheta=5.62^{\circ}$ and $\delta \varphi=7.50^{\circ}$ with an auto-correlation of 0.69 . The local statistics for this patch are as follows: $A d_{r} / A d_{h}=1.16 ; S t_{r} / S t_{h}=0.65 ; S t_{h} / A d_{h}=2.10 ; S t / A d=1.47 ; \mathcal{P} / \mathcal{T}=1.07 ;$ and $\left\|u_{r}\right\| /\left\|\vec{u}_{h}\right\|=1.62$ 
shows the proportion of outer boundary patches for which roots were detected for each dynamo model. Dynamo models with stronger magnetic advection effects exhibit more distortion from the outer boundary to depth. Indeed, dynamo model 9 has the lowest percentage of roots and the highest $R m$ while dynamo models 1 and 7 have the highest percentage of roots and a fairly low $R m$. In addition, we separated the percentage of detected roots by patch type. We note that in the strong advection dynamo model 9, the percentage of detected $\mathrm{HN}$ roots is lower than the percentage of detected LN and LR roots, whereas in the weaker advection dynamo models 1 and 7, the percentage of $\mathrm{HN}$ roots is either comparable to or larger than that of LN and LR roots.

In general, the mid-depth roots appear with some shift in latitude and longitude with respect to the location of the outer boundary patches. Table 4 shows the average a

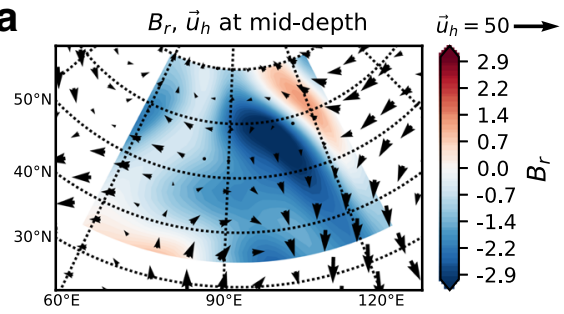

C

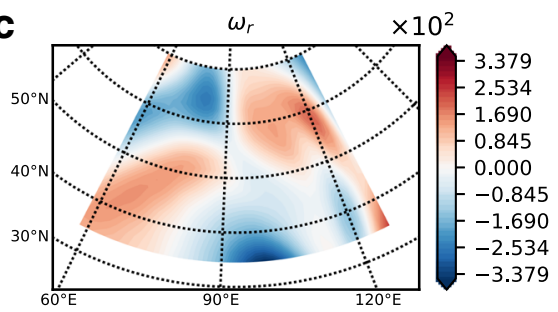

e

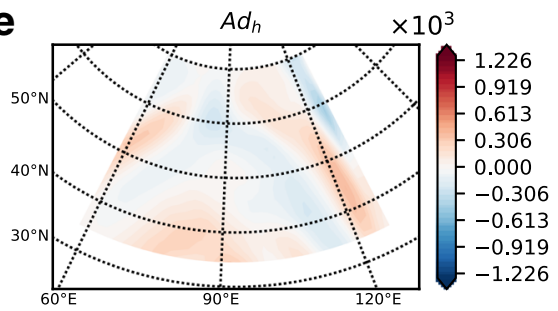

g

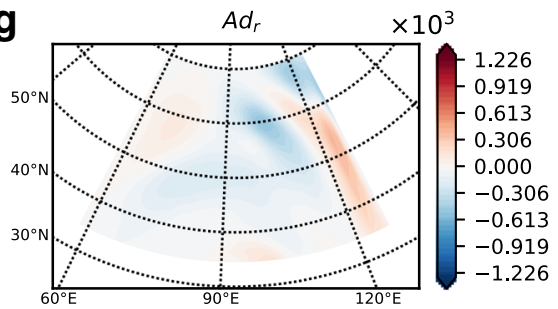

i

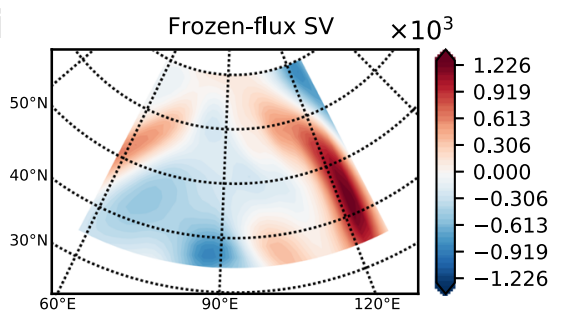

b

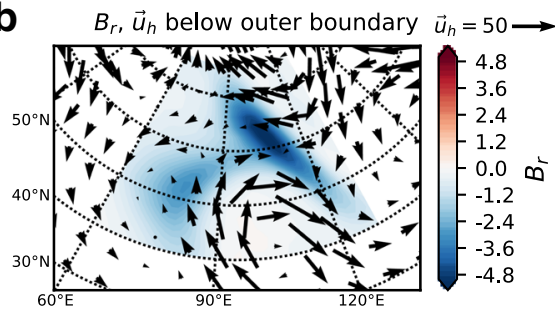

d

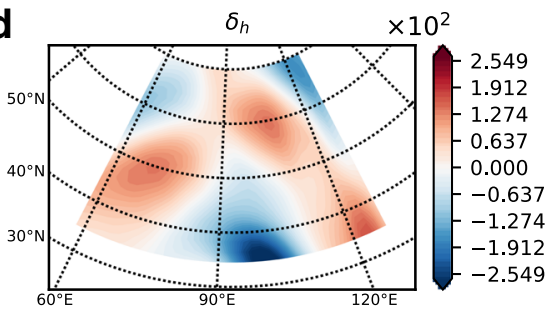

f

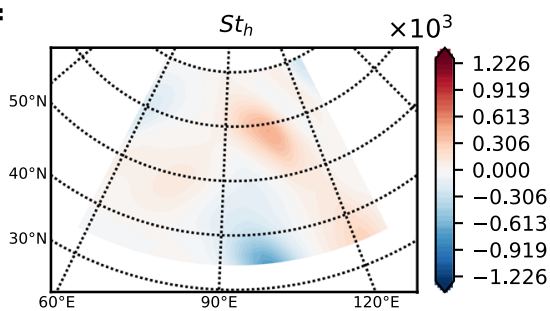

h

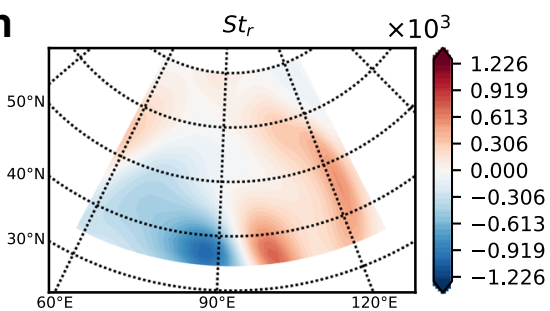

j

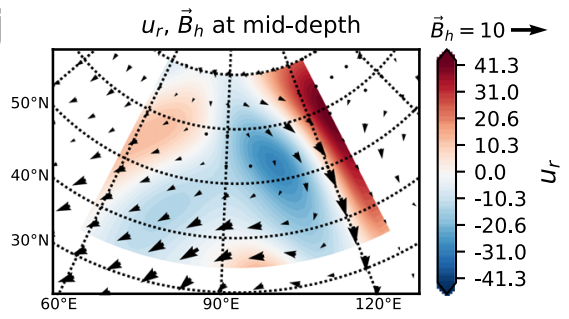

Fig. 9 As in Fig. 6 for another intense high-latitude normal polarity magnetic flux patch (HN). This patch presents a dominant $S t_{r}$ in dynamo model 1. The mid-depth patch in $\mathbf{a}$ is shifted with respect to the outer boundary patch in $\mathbf{b}$ by $\delta \vartheta=5.62^{\circ}$ and $\delta \varphi=-1.88^{\circ}$ with an auto-correlation of 0.53 . The local statistics for this patch are as follows: $A d_{r} / A d_{h}=0.87 ; S t_{r} / S t_{h}=2.42 ; S t_{h} / A d_{h}=0.98 ; S t / A d=1.74 ; \mathcal{P} / \mathcal{T}=0.57 ;$ and $\left\|u_{r}\right\| /\left\|\vec{u}_{h}\right\|=1.00$ 
latitudinal shift $\delta \vartheta$ of the identified mid-depth roots. In some cases, the shift is rather close to the $10.7^{\circ}$ value expected from the tangential cylinder rim effect, e.g., in dynamo models 4 and 8 with tangential advection dominance and in dynamo model 5 with radial stretching dominance. Overall, the positive $\delta \vartheta$ for HN (Table 4 and Figs. 6, 7, 8, 9, and 10) is consistent with some tangent cylinder rim effect at high latitudes. In contrast, the generally lower $\delta \vartheta$ for LN and LR is suggestive of more plume-like dynamics at low latitudes. Lower standard deviations were found for $\mathrm{HN}$, supporting the robustness of lower latitude roots for these features. In contrast, the values for $L N$ and $L R$ are more dispersed.

In some cases, small $\delta \varphi$ values were found (Figs. 6, 9, and 10). This is consistent with both a tangent cylinder rim effect and a plume-like effect. However, the larger $\delta \varphi$ values in other cases (Figs. 7 and 8 ) are indicative of more complex kinematics.
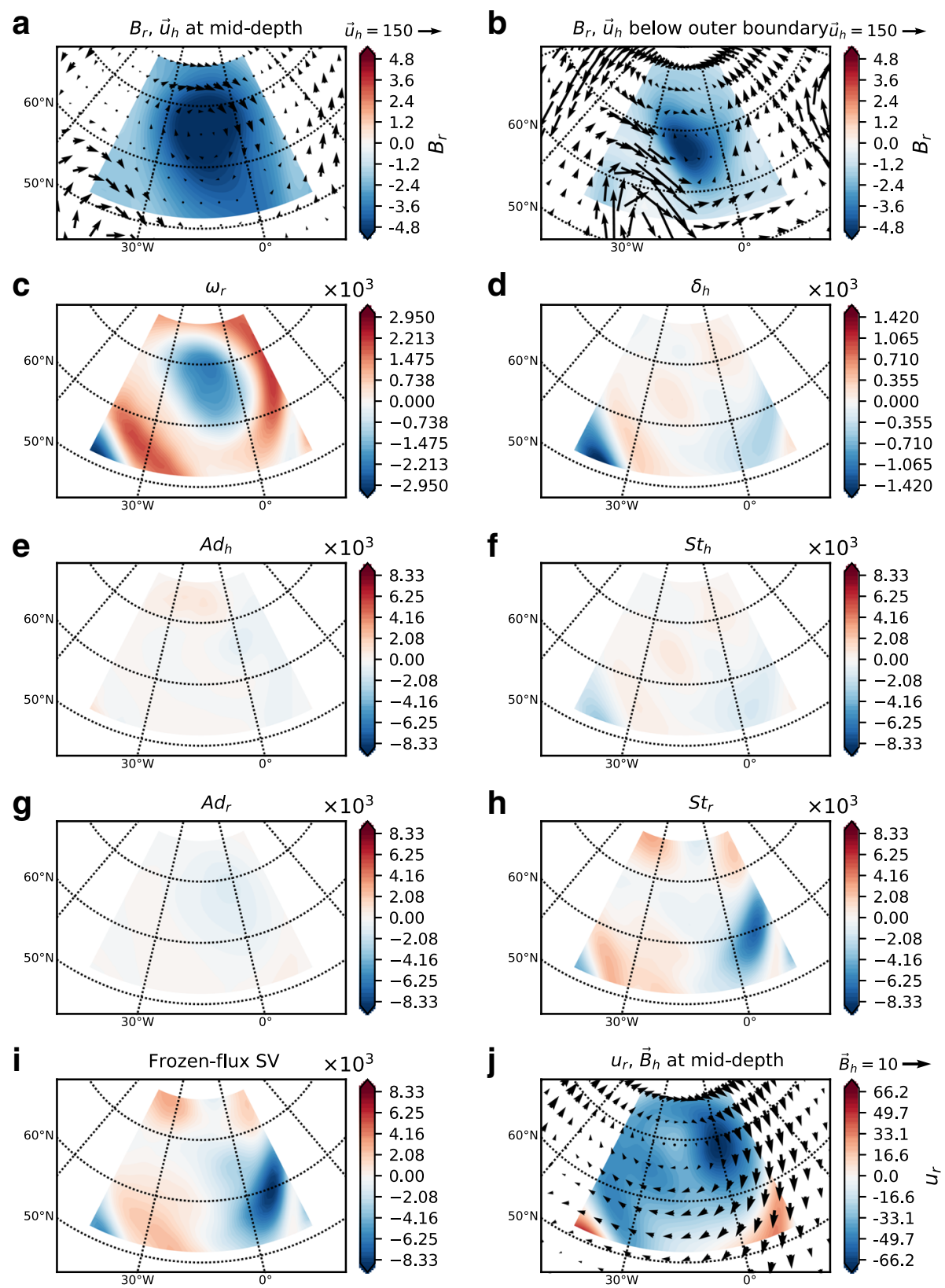

Fig. 10 As in Fig. 6 for another intense high-latitude normal polarity magnetic flux patch (HN). This patch presents a dominant $S t_{r}$ in dynamo model 7. The mid-depth patch in $\mathbf{a}$ is shifted with respect to the outer boundary patch in $\mathbf{b}$ by $\delta \vartheta=5.62^{\circ}$ and $\delta \varphi=0^{\circ}$ with an auto-correlation of 0.80 . The local statistics for this patch are as follows: $A d_{r} / A d_{h}=1.26 ; S t_{r} / S t_{h}=2.77 ; S t_{h} / A d_{h}=2.52 ; S t / A d=4.86 ; \mathcal{P} / \mathcal{T}=0.25 ;$ and $\left\|u_{r}\right\| /\left\|\vec{u}_{h}\right\|=0.71$ 
The local statistics for each dynamo model and root type are given in Table 5. The statistics for the roots are similar to the global statistics, with a dominance of $A d_{h}$ over $A d_{r}$ and of $S t_{r}$ over $S t_{h}$. In the HN roots, the contribution of the total stretching to the frozen-flux SV is larger than the total advection contribution despite the low and moderate values of $\mathcal{P} / \mathcal{T}$ and $\left\|u_{r}\right\| /\left\|\vec{u}_{h}\right\|$, respectively. In contrast, the roots of $L N$ and $L R$ patches exhibit a larger advection contribution to the frozen-flux SV. Also, the poloidal part of the flow is stronger in LN and LR than in HN.
Figure 6 is an example of a very clear root at middepth from dynamo model 1 . The outer boundary HN patch in Fig. 6b is highly auto-correlated (0.68) with the deep structure detected in Fig. 6a. The flow pattern also exhibits some similarities between mid-depth and the outer boundary. The tangential flow is dominantly toroidal, and the radial component of the flow is only 0.62 of the tangential component. Significant fieldflow alignment seen in Fig. 6a produces a relatively weak $A d_{h}$ (Fig. 6e) in some parts of the area. However, $A d_{h}$ is

Table 5 Local statistics at mid-depth: dynamo models time average and standard deviation values for each patch type (PT)

\begin{tabular}{|c|c|c|c|c|c|c|c|}
\hline Model & PT & $A d_{r} / A d_{h}$ & $S t_{r} / S t_{h}$ & $S t_{h} / A d_{h}$ & St/Ad & $\mathcal{P} / \mathcal{T}$ & $\left\|u_{r}\right\| /\left\|\vec{u}_{h}\right\|$ \\
\hline & $\mathrm{HN}$ & $0.69 \pm 0.29$ & $1.31 \pm 0.50$ & $0.65 \pm 0.28$ & $0.90 \pm 0.46$ & $0.36 \pm 0.12$ & $0.74 \pm 0.24$ \\
\hline \multirow[t]{3}{*}{1} & LN & $0.46 \pm 0.00$ & $2.87 \pm 0.00$ & $0.20 \pm 0.00$ & $0.56 \pm 0.00$ & $0.27 \pm 0.00$ & $0.45 \pm 0.00$ \\
\hline & LR & $0.77 \pm 0.38$ & $0.84 \pm 0.21$ & $0.63 \pm 0.32$ & $0.71 \pm 0.28$ & $0.41 \pm 0.14$ & $0.97 \pm 0.21$ \\
\hline & $\mathrm{HN}$ & $0.71 \pm 0.29$ & $1.49 \pm 0.67$ & $0.52 \pm 0.37$ & $0.81 \pm 0.46$ & $0.37 \pm 0.16$ & $0.82 \pm 0.32$ \\
\hline \multirow[t]{3}{*}{2} & LN & $0.55 \pm 0.11$ & $0.77 \pm 0.18$ & $0.56 \pm 0.02$ & $0.67 \pm 0.11$ & $0.35 \pm 0.05$ & $0.98 \pm 0.08$ \\
\hline & $L R$ & $0.55 \pm 0.28$ & $1.15 \pm 0.51$ & $0.38 \pm 0.15$ & $0.55 \pm 0.18$ & $0.37 \pm 0.15$ & $0.90 \pm 0.40$ \\
\hline & $\mathrm{HN}$ & $0.61 \pm 0.27$ & $1.53 \pm 0.80$ & $0.42 \pm 0.13$ & $0.63 \pm 0.26$ & $0.32 \pm 0.11$ & $0.72 \pm 0.18$ \\
\hline \multirow[t]{3}{*}{3} & LN & - & - & - & - & - & - \\
\hline & $L R$ & $2.23 \pm 1.69$ & $0.87 \pm 0.44$ & $0.99 \pm 0.72$ & $0.56 \pm 0.25$ & $1.26 \pm 0.63$ & $1.65 \pm 1.29$ \\
\hline & $\mathrm{HN}$ & $0.77 \pm 0.33$ & $1.53 \pm 0.82$ & $0.92 \pm 0.38$ & $1.35 \pm 0.66$ & $0.33 \pm 0.08$ & $0.80 \pm 0.24$ \\
\hline \multirow[t]{3}{*}{4} & LN & - & - & - & - & - & - \\
\hline & $L R$ & - & - & - & - & - & - \\
\hline & $\mathrm{HN}$ & $0.68 \pm 0.24$ & $1.29 \pm 0.45$ & $1.02 \pm 0.38$ & $1.50 \pm 0.91$ & $0.40 \pm 0.12$ & $0.71 \pm 0.20$ \\
\hline \multirow[t]{3}{*}{5} & LN & - & - & - & - & - & - \\
\hline & LR & - & - & - & - & - & - \\
\hline & $\mathrm{HN}$ & $0.65 \pm 0.30$ & $1.44 \pm 0.79$ & $0.54 \pm 0.25$ & $0.81 \pm 0.33$ & $0.37 \pm 0.17$ & $0.95 \pm 0.50$ \\
\hline \multirow[t]{3}{*}{6} & LN & $0.52 \pm 0.18$ & $1.10 \pm 0.13$ & $0.55 \pm 0.09$ & $0.54 \pm 0.24$ & $1.18 \pm 0.62$ & $0.62 \pm 0.08$ \\
\hline & $L R$ & $0.78 \pm 0.14$ & $0.91 \pm 0.41$ & $0.71 \pm 0.15$ & $1.03 \pm 0.26$ & $0.53 \pm 0.14$ & $1.21 \pm 0.20$ \\
\hline & $\mathrm{HN}$ & $0.78 \pm 0.19$ & $1.37 \pm 0.47$ & $1.39 \pm 0.50$ & $1.75 \pm 1.03$ & $0.40 \pm 0.21$ & $0.82 \pm 0.28$ \\
\hline \multirow[t]{3}{*}{7} & LN & $0.48 \pm 0.22$ & $1.44 \pm 0.70$ & $0.53 \pm 0.31$ & $0.99 \pm 0.48$ & $0.24 \pm 0.01$ & $0.66 \pm 0.19$ \\
\hline & $L R$ & $0.56 \pm 0.00$ & $1.25 \pm 0.00$ & $0.38 \pm 0.00$ & $0.51 \pm 0.00$ & $0.33 \pm 0.00$ & $0.66 \pm 0.00$ \\
\hline & $\mathrm{HN}$ & $0.70 \pm 0.20$ & $1.33 \pm 0.58$ & $1.18 \pm 0.49$ & $1.68 \pm 0.92$ & $0.47 \pm 0.17$ & $0.85 \pm 0.22$ \\
\hline \multirow[t]{3}{*}{8} & LN & - & - & - & - & - & - \\
\hline & $L R$ & $0.68 \pm 0.00$ & $1.42 \pm 0.00$ & $0.43 \pm 0.00$ & $0.80 \pm 0.00$ & $0.41 \pm 0.00$ & $0.59 \pm 0.00$ \\
\hline & $\mathrm{HN}$ & $0.64 \pm 0.21$ & $1.03 \pm 0.47$ & $0.64 \pm 0.26$ & $0.77 \pm 0.27$ & $0.35 \pm 0.12$ & $1.18 \pm 0.79$ \\
\hline \multirow[t]{3}{*}{9} & LN & $0.74 \pm 0.17$ & $0.99 \pm 0.39$ & $0.71 \pm 0.00$ & $1.12 \pm 0.20$ & $0.52 \pm 0.01$ & $1.14 \pm 0.04$ \\
\hline & $L R$ & $0.87 \pm 0.34$ & $0.57 \pm 0.15$ & $0.80 \pm 0.51$ & $1.01 \pm 0.64$ & $0.45 \pm 0.05$ & $1.37 \pm 0.16$ \\
\hline & $\mathrm{HN}$ & $0.70 \pm 0.28$ & $1.40 \pm 0.65$ & $0.77 \pm 0.47$ & $1.10 \pm 0.75$ & $0.37 \pm 0.15$ & $0.81 \pm 0.33$ \\
\hline \multirow[t]{2}{*}{$\bar{x}$} & LN & $0.56 \pm 0.19$ & $1.28 \pm 0.73$ & $0.54 \pm 0.21$ & $0.80 \pm 0.36$ & $0.54 \pm 0.46$ & $0.80 \pm 0.26$ \\
\hline & LR & $0.83 \pm 0.76$ & $0.98 \pm 0.43$ & $0.58 \pm 0.39$ & $0.68 \pm 0.33$ & $0.50 \pm 0.36$ & $1.04 \pm 0.57$ \\
\hline
\end{tabular}


the dominant SV mechanism due to other highly advective regions where the flow is nearly perpendicular to the $B_{r}$-isolines. The contribution of $A d_{r}$ is low (see Fig. $6 \mathrm{~g}$ ). $S t_{r}$ is the second dominant mechanism (Fig. 6h), with a strong feature due to a strong meridional magnetic field $B_{\theta}$ sheared by the edge of an intense descending flow (Fig. 6j). Also, this $S t_{r}$ feature is located near an intense tangentially diverging structure (Fig. $6 \mathrm{~d}$ ), but because the local radial field is weak, no significant $S t_{h}$ contribution appears there (Fig. 6f). In this root, the total stretching SV amounts to 0.78 of the total advective SV.

The root shown in Fig. 7 is an example of a strong $A d_{h}$ contribution from dynamo model 3 . This mid-depth patch is highly auto-correlated with the $\mathrm{HN}$ patch detected at the outer boundary (Fig. 7b), shifted by $\delta \vartheta=1.88^{\circ}$ (i.e. to lower latitudes) and $\delta \varphi=-9.38^{\circ}$ (i.e. to the west). Westward flow roughly perpendicular to the $B_{r^{-}}$isolines (Fig. 7a) produces a characteristic bipolar SV pattern centered at the center of the patch (Fig. 7e) with its axis parallel to the direction of the flow (Amit 2014; Peña et al. 2016; Livermore et al. 2017). The low value of $S t_{h}$ is due to the low correlation between $\delta_{h}$ (Fig. $7 \mathrm{~d}$ ) and the $B_{r}$ feature (Fig. 7a). The intense $u_{r}$ in Fig. $7 \mathrm{j}$ is not well correlated with the $B_{r}$ feature in Fig. 7a, resulting in a weak $A d_{r}$ (Fig. 7g). In addition, the significant field-flow alignment between $u_{r}$ and $\vec{B}_{h}$ (Fig. 7j) results in a weak $S t_{r}$ (Fig. 7h).

Figure 8 shows a mid-depth root with $S t_{h}$ dominance from dynamo model 2. The high auto-correlation (0.69) between the deep structure detected in Fig. $8 \mathrm{a}$ and the outer boundary $\mathrm{HN}$ patch in Fig. $8 \mathrm{~b}$ is very clear. The root is shifted by $\delta \vartheta=5.62^{\circ}$ (i.e., to lower latitudes) and $\delta \varphi=7.50^{\circ}$ (i.e., to the east). The tangential flow has a large field-aligned component which produces a weak $A d_{h}$ (Fig. 8e). In contrast, the overlap of the tangential divergence/convergence structures (Fig. 8d) with the $B_{r}$ root (Fig. 8a) produces the strong $S t_{h}$ contribution in Fig. 8f. For example, the northeast peak of $B_{r}$ (Fig. 8a) is dispersed by tangential divergence (a positive $\delta_{h}$ in the same area in Fig. 8d) giving a strong negative $S t_{h}$ there (Fig. 8f). In addition, both $A d_{r}$ and $S t_{r}$ are locally strong due to the strong descending flow in the northern part (Fig. 8j). The $A d_{r}$ structure (Fig. 8g) appears near the peak of $u_{r}$ where the radial field is advected from above, whereas the $S t_{r}$ bipolar structure (Fig. 8h) appears at the edges of the $u_{r}$ structure where $B_{\theta}$ is sheared by a variable convective flow in the north-south direction (Fig. 8j). In this root, the poloidal flow is slightly larger than the toroidal and the radial flow is significantly larger than the tangential. Overall, in this patch, stretching mechanisms dominate over advection mechanisms in the frozen-flux SV by a significant factor of 1.47 .

Mid-depth roots dominated by $A d_{r}$ are rare (Table 6). On average, $A d_{r}$ is significantly weaker than $A d_{h}$ (Table 5).
Table 6 Dominant SV mechanisms: portion of the dominant SV mechanisms (in percentage) in all the identified roots for each dynamo model

\begin{tabular}{lllll}
\hline Model & $A d_{h}$ & $S t_{h}$ & $A d_{r}$ & $S t_{r}$ \\
\hline 1 & 71.4 & 7.1 & 7.1 & 14.3 \\
2 & 80.9 & 4.3 & 10.6 & 4.3 \\
3 & 80 & 0 & 20 & 0 \\
4 & 31.3 & 18.8 & 6.3 & 43.8 \\
5 & 35 & 20 & 0 & 45 \\
6 & 76 & 4 & 8 & 12 \\
7 & 17.4 & 8.7 & 0 & 73.9 \\
8 & 41.7 & 8.3 & 0 & 50 \\
9 & 87.5 & 12.5 & 0 & 0 \\
\hline
\end{tabular}

Over all nine dynamo models for $\mathrm{HN}$ patches, the ratio $A d_{r} / A d_{h}$ has a rather narrow range of 0.61-0.78.

Finally, the root shown in Fig. 9 presents a dominant $S t_{r}$ contribution from dynamo model 1 . This root is again well auto-correlated with the $\mathrm{HN}$ patch detected at the outer boundary (Fig. $9 \mathrm{~b}$ ), shifted by $\delta \vartheta=5.62^{\circ}$ (again to lower latitudes) and $\delta \varphi=-1.88^{\circ}$ (i.e., to the west). It is located in the middle of a flow saddle (Fig. 9c) that generates a relatively weak $A d_{h}$ (Fig. 9e). This flow is predominantly toroidal and with comparable radial and tangential components. The tangential divergence (Fig. 9d) overlaps with the intense $B_{r}$, resulting in some $S t_{h}$ contribution (Fig. 9f). However, the strong total stretching SV mostly originates from the dominant $S t_{r}$ contribution (Fig. 9h) due to the strong azimuthal field $B_{\varphi}$ in the southern part (Fig. 9j). Note that in the eastern part, strong $u_{r}$ and $B_{\theta}$ features yield a weak $S t_{r}$ due to the north-south orientation of the radial flow structure. Similar $S t_{r}$ dominance can also be found in lower $E$ dynamo models. Figure 10 focuses on an HN patch from our lowest $E$ and $P m$ dynamo model 7 . Although the tangential field is in general nearly parallel to the $u_{r}$ isolines (Ferraro's law; see Aubert 2005), small deviations from this field-flow alignment suffice to produce intense $S t_{r}$ contributions at the edges of a descending flow structure where the tangential field is strong.

In general, we found a predominance of $A d_{h}$ (more than $\sim 70 \%$ of the studied roots in five dynamos; see Table 6 ). The other four dynamos exhibit a larger number of roots with $S t_{r}$ dominance, but only in dynamo model 7 the number of roots with $S t_{r}$ dominance is significantly larger than the other mechanisms. Radial advection is the least dominant mechanism.

\section{Parameter dependence}

In order to examine quantitatively the dependence of the statistical measures on the non-dimensional control parameters, we used a generic power law (25) as in 
Peña et al. (2016). Power law fits were applied for global and local measures. Significantly small powers were omitted. Also, we assigned the same value to powers with similar values. Finally, we approximated powers by discrete values. These steps were monitored using the relative misfit (26). The resulting power laws are shown in Table 7.

Figure 11 shows the parameter dependence of $A d_{r} / A d_{h}$ and $S t_{r} / S t_{h}$. Globally, relative radial advection in the dynamo models increases with increasing convection but decreases when rotation increases (Fig. 11a). In the roots of high-latitude normal polarity patches $(\mathrm{HN})$, we find an opposite behavior: relative radial advection decreases with increasing convection but increases when rotation increases (Fig. 11b). In both cases, global and local, the dependence is stronger on $E$. Global relative radial stretching increases with increasing rotation but decreases when convection and electrical conductivity increase. In this case, the dependence is strongest on $E$ (Fig. 11c). Relative radial stretching on $\mathrm{HN}$ increases with increasing rotation but decreases when convection and electrical conductivity increase. This is in qualitative agreement with the global case. Relative radial stretching exhibits a strong dependence on $\mathrm{Ra}$ in $\mathrm{HN}$ roots (Fig. 11d).

We also compared the parameter dependence of $S t_{h} / A d_{h}$ and $S t / A d$ (Fig. 12). Globally, relative tangential stretching is mostly influenced by $P m$, decreasing with increasing electrical conductivity (Fig. 12a). In HN roots, relative tangential stretching increases with increasing rotation but decreases when convection and electrical conductivity increase. The dependence is strongest on $E$ (Fig. 12b). Both global and local total relative stretching also increase with increasing rotation and decrease when convection and electrical conductivity increase (Fig. 12c, d). However, the global $S t / A d$ dependence is strongest on Pm (Fig. 12c) whereas in HN roots, St/Ad exhibits a strong dependence on $E$ (Fig. 12d).

Finally, Fig. 13 shows the parameter dependence of $\mathcal{P} / \mathcal{T}$ and $\left\|u_{r}\right\| /\left\|\vec{u}_{h}\right\|$. Globally, relative poloidal flow decreases with increasing rotation but increases when convection increases. The dependence is strongest on $E$ (Fig. 13a). In contrast, relative poloidal flow in $\mathrm{HN}$ roots is equally influenced by $E$ and $P m$, increasing when rotation and electrical conductivity increase (Fig. 13b). Similar to global relative poloidal flow, global relative radial flow exhibits a strong dependence on $E$, decreasing with increasing rotation and with decreasing convection (Fig. 13c). This similarity is expected since the radial flow is strictly poloidal. Relative radial flow in HN roots increases when convection and electrical conductivity increase, with a strongest dependence on $P m$ (Fig. 13d).

\section{Discussion}

The radial magnetic induction equation at the $\mathrm{CMB}$ in the frozen-flux limit (11) has been thoroughly explored in the context of core flow inversions from geomagnetic SV data (e.g. Bloxham and Jackson 1991; Holme 2015). According to this equation, tangential advection and tangential stretching mechanisms $\left(A d_{h}\right.$ and $\left.S t_{h}\right)$ induce the SV at

Table 7 Parameter dependence: power law fits and relative misfit $\sigma_{r}$

\begin{tabular}{|c|c|c|c|c|c|}
\hline Quantity & Type & Mid-shell & $\sigma_{r}$ & $\delta \sigma_{r}$ & Outer boundary \\
\hline \multirow{3}{*}{$A d_{r} / A d_{h}$} & Global & $0.738 \cdot\left(E^{2} \cdot R a\right)^{\frac{1}{8}}$ & 0.029 & 0.004 & - \\
\hline & $\mathrm{HN}$ & $0.580 \cdot\left(E^{2} \cdot R a\right)^{-\frac{1}{11}}$ & 0.042 & 0.000 & - \\
\hline & Global & $2.081 \cdot\left(E^{3} \cdot R a^{2} \cdot P m\right)^{-\frac{1}{12}}$ & 0.054 & 0.007 & - \\
\hline \multirow[t]{2}{*}{$S t_{r} / S t_{h}$} & $\mathrm{HN}$ & $2.982 \cdot\left(E \cdot R a^{2} \cdot P m\right)^{-\frac{1}{28}}$ & 0.080 & 0.000 & - \\
\hline & Global & $0.628 \cdot P m^{-\frac{1}{4}}$ & 0.071 & 0.004 & $2.996 \cdot\left(E \cdot R a \cdot P m^{3}\right)^{-\frac{1}{6}}$ \\
\hline \multirow[t]{2}{*}{$S t_{h} / A d_{h}$} & $\mathrm{HN}$ & $0.765 \cdot\left(E^{6} \cdot R a^{3} \cdot P m^{5}\right)^{-\frac{1}{11}}$ & 0.140 & 0.005 & $10.489 \cdot\left(E \cdot R a \cdot P m^{2}\right)^{-\frac{1}{3}}$ \\
\hline & Global & $1.666 \cdot\left(E^{4} \cdot R a^{3} \cdot P m^{6}\right)^{-\frac{1}{24}}$ & 0.036 & 0.004 & $2.996 \cdot\left(E \cdot R a \cdot P m^{3}\right)^{-\frac{1}{6}}$ \\
\hline \multirow[t]{2}{*}{ St/Ad } & $\mathrm{HN}$ & $0.425 \cdot\left(E^{9} \cdot R a^{4} \cdot P m^{3}\right)^{-\frac{1}{13}}$ & 0.184 & 0.016 & $10.489 \cdot\left(E \cdot R a \cdot P m^{2}\right)^{-\frac{1}{3}}$ \\
\hline & Global & $0.302 \cdot\left(E^{3} \cdot R a^{2}\right)^{\frac{1}{16}}$ & 0.035 & 0.002 & $0.319 \cdot P m^{\frac{1}{6}}$ \\
\hline \multirow[t]{2}{*}{$\mathcal{P} / \mathcal{T}$} & $\mathrm{HN}$ & $0.105 \cdot\left(E^{-1} \cdot P m\right)^{\frac{1}{8}}$ & 0.089 & 0.002 & $0.261 \cdot P m^{\frac{1}{6}}$ \\
\hline & Global & $0.556 \cdot\left(E^{3} \cdot R a^{2}\right)^{\frac{1}{16}}$ & 0.025 & 0.005 & - \\
\hline$\left\|u_{r}\right\| /\left\|\vec{u}_{h}\right\|$ & $\mathrm{HN}$ & $0.117 \cdot\left(R a^{3} \cdot P m^{5}\right)^{\frac{1}{20}}$ & 0.078 & 0.003 & - \\
\hline
\end{tabular}

The fit deterioration is defined by $\delta \sigma_{r}=\sigma_{r}-\sigma_{r 0}$ where $\sigma_{r o}$ is the initial best fit relative misfit. Quantities are the same as in Table 2. Also given are the power laws for the outer boundary (Peña et al. 2016) 

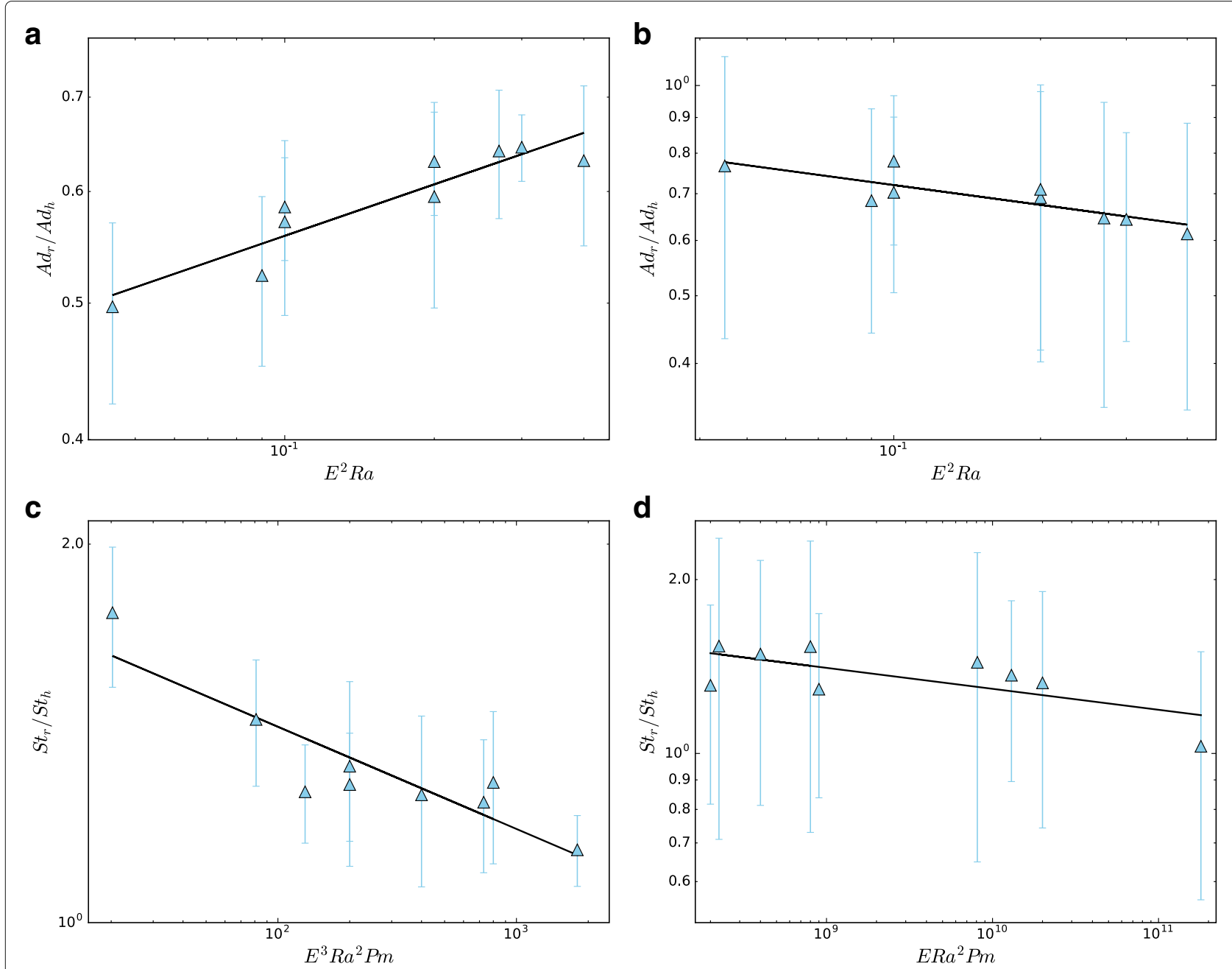

Fig. 11 Parameter dependence of $A d_{r} / A d_{h}$ and $S t_{r} / S t_{h}$ rms ratios. Each point represents $\mathbf{a}, \mathbf{c}$ global mean values and $\mathbf{b}$, $\mathbf{d} H N$ mean values of each dynamo simulation. Standard deviations are denoted by error bars which represent time dependence

the top of the core. The much-less explored radial induction equation away from the boundaries where $u_{r} \neq 0$ includes additional radial advection and radial stretching mechanisms $\left(A d_{r}\right.$ and $S t_{r}$; see Fig. 1) which lead to richer kinematic scenarios.

At mid-shell, global tangential advection dominates the $\mathrm{SV}$ in all dynamo models. Total stretching SV varies between two thirds of total advection to comparable, whereas the toroidal flow is $2-3$ times larger than the tangential poloidal flow (Table 2). Similar results were reported by Peña et al. (2016) at the top of the shell. Thus, the contribution of stretching mechanisms to the SV at mid-depth is more significant than expected based on the relative strength of the poloidal flow at the top of the shell.

The radial magnetic field is concentrated in robust intense flux patches, as observed at the top of the shell of numerical dynamos as well as in the geomagnetic field models on the CMB (Christensen et al. 2010). In contrast, we found that at depth, the radial magnetic field is more distributed over the spherical surface (Fig. 5). This weaker flux concentration at mid-depth renders challenging the identification of deep roots of the intense flux patches at the top of the shell. Nevertheless, proper accounting for various image distortion effects led us to identify such roots.

Different portions of detected roots were found for the different dynamo models (see Table 3). The number of identified roots is roughly inversely proportional to the magnetic Reynolds number $R m$. Stronger magnetic mixing effects at larger $R m$ correspond to more vigorous magnetic advection effects between the top and mid-shell, which distort the patches and reduce the possibility of identifying their roots. The identified mid-depth roots were classified by polarity and by latitude according to their correlated outer boundary structures. Roots of highlatitude intense normal flux patches $(\mathrm{HN})$ were identified 

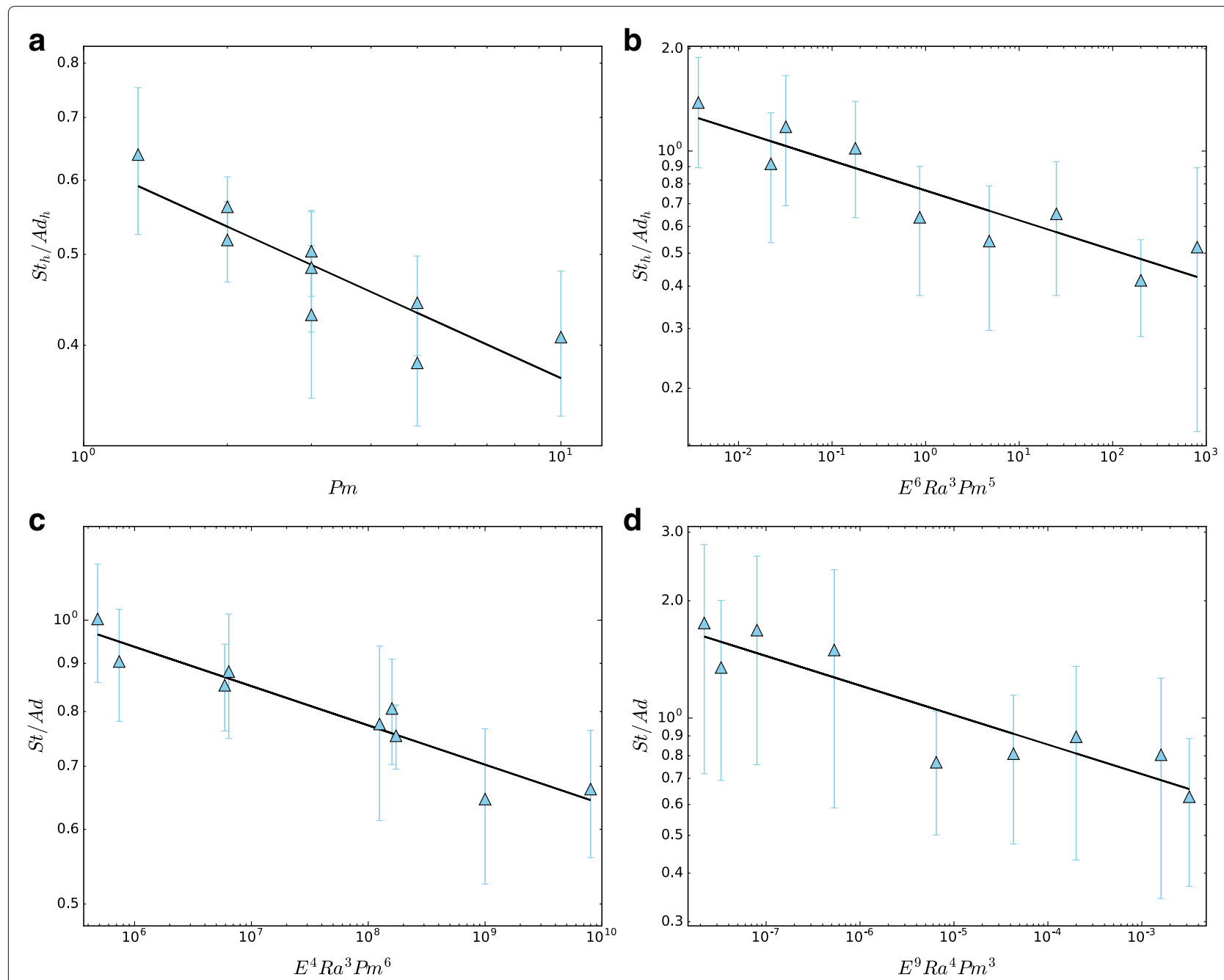

Fig. 12 As in Fig. 11 for $S t_{h} / A d_{h}$ and St/Ad rms ratios

in all dynamo models, but the fraction of $\mathrm{HN}$ detected roots is also dependent on the strength of magnetic advection at each dynamo model. Stronger advection models exhibit a lower $\mathrm{HN}$ detection than that of the roots of lowlatitude normal and reversed flux patches (LN and LR, respectively), whereas in weaker advection models, the $\mathrm{HN}$ detection is either comparable to or larger than that of LN and LR (Table 3).

The roots exhibit a position shift with respect to the location of the outer boundary patches. Generally, the latitudinal shift $\delta \vartheta>0$, i.e., the roots are at lower latitudes than the outer boundary patches, in qualitative agreement with a tangent cylinder rim effect (Fig. 2). However, the mean latitudinal shift is lower than expected from a pure tangent cylinder rim effect $\left(\delta \vartheta<10.7^{\circ}\right.$; see Table 4$)$, indicating a mixed effect of a tangent cylinder rim and plume-like dynamics.

Relative stretching varies for different root types. In the $\mathrm{HN}$ roots, stretching dominates the SV in field-aligned flow regions where advection is not sufficiently effective, despite $A d_{h}$ being the single dominant SV mechanism (Table 6). On average in HN, total stretching SV exceeds total advection by a factor of 1.1, with the poloidal flow being only 0.37 of the toroidal flow (Table 5 ). These results are consistent with those found in the HN outer boundary patches reported by Peña et al. (2016). The contribution of radial stretching is significant for total stretching SV dominance at depth. In contrast, the structures at low latitudes (LN and LR) exhibit lower values of total relative stretching and higher values of relative poloidal flow.

At mid-shell, the ratio of global tangential stretching over tangential advection increases with decreasing electrical conductivity. In $\mathrm{HN}$ roots, relative tangential stretching increases with decreasing $E, R a$, and $P m$. The strongest dependence is on $E$, but $P m$ also exhibits a strong influence (Fig. 12a, b). The ratio of total stretching over total advection also increases with decreasing $E, R a$, and $P m$ in both the global and $\mathrm{HN}$ roots 

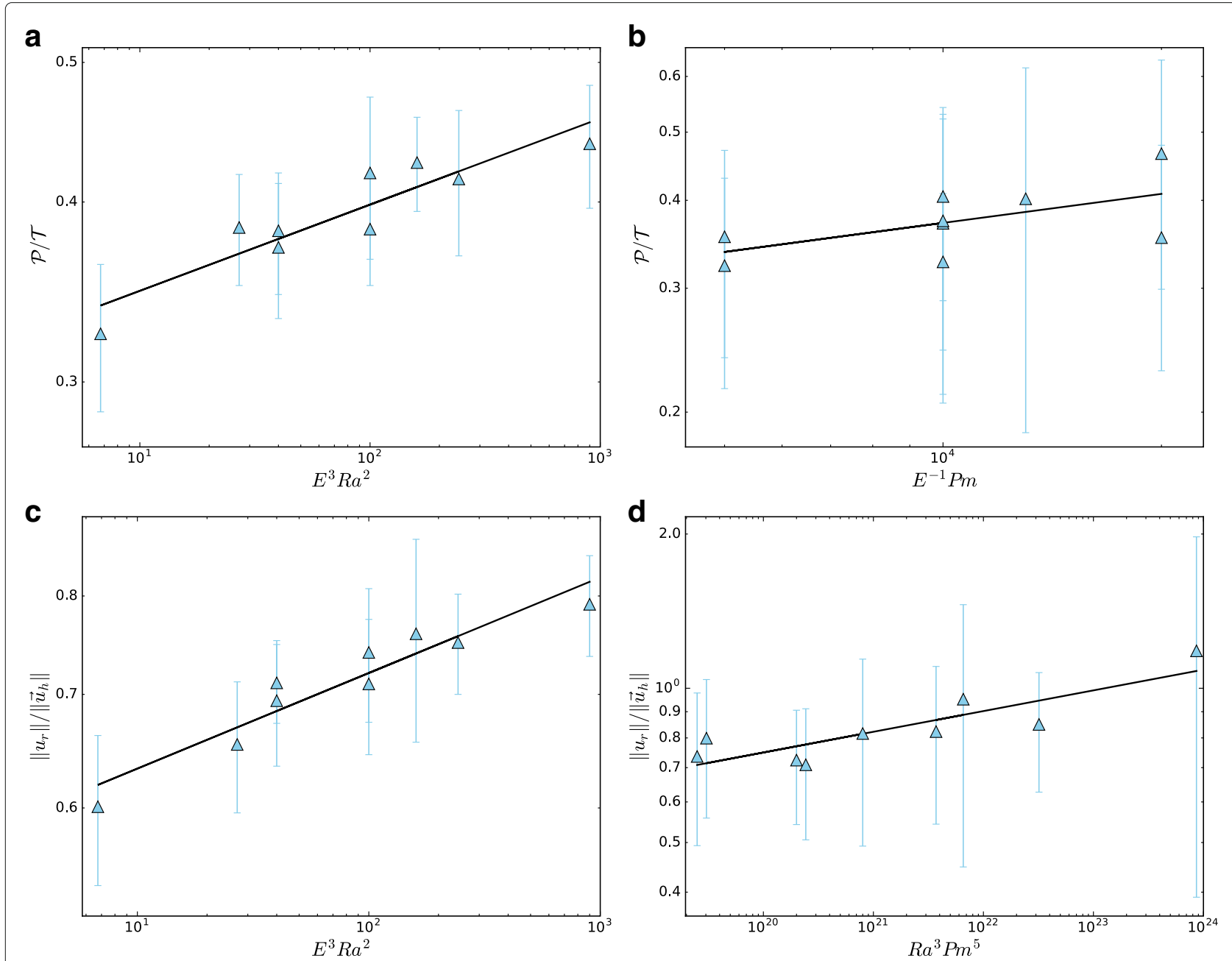

Fig. 13 As in Fig. 11 for $\mathcal{P} / \mathcal{T}$ and $\left\|u_{r}\right\| /\left\|\vec{u}_{h}\right\|$ rms ratios

cases. Globally, the $S t / A d$ dependence is strongest on $P m$ while the dependence in the $\mathrm{HN}$ roots is strongest on $E$ (Fig. 12c, d). These behaviors are in qualitative agreement with the decrease with $E, R a$, and $P m$ found by Peña et al. (2016) for the ratio of stretching over advection on the outer boundary (see Table 7).

The relative global poloidal flow at mid-shell is strongly influenced by $E$, increasing with increasing $E$ and $R a$. In contrast, the relative poloidal flow in the $\mathrm{HN}$ roots increases with increasing $E$ and decreasing $P m$ (Fig. 13a, b). These results contradict the behavior on the outer boundary, where both global and $\mathrm{HN} \mathcal{P} / \mathcal{T}$ only depend on $P m$, increasing with increasing $P m$ (Peña et al. 2016). The parameter dependence of the ratio of global radial flow over global tangential flow is practically identical to that of $\mathcal{P} / \mathcal{T}$. The relative radial flow at $\mathrm{HN}$ also has the strongest dependence on $P m$, increasing with increasing $R a$ and $P m$ (Fig. 13c, d).

It is worth elaborating on the radial stretching SV mechanism because it contributes significantly at mid-shell while it is by definition absent at the outer boundary where the radial flow vanishes. Of the four radial field SV mechanisms at depth (Fig. 1), radial stretching is the only dynamo mechanism (Oslon et al. 1999, Aubert et al. 2008b). When the tangential magnetic field lines $\vec{B}_{h}$ are perpendicular to the $u_{r}$-isolines, strong $S t_{r}$ structures are produced (as in Fig. $9 \mathrm{~h}, \mathrm{j}$ ). These strong $S t_{r}$ structures are characterized by an intense tangential field residing at the edges of radial plumes where the tangential gradient of $u_{r}$ is large. As illustrated in Fig. 1d, this process transforms a (tangential) toroidal magnetic field into a (radial) poloidal one.

Takahashi and Shimizu (2012) performed a detailed local analysis of the field generation process in numerical dynamos. They found four typical stretching scenarios, two shallow and two deep. One deep stretching mechanism is associated with downwellings inside columnar vortices, which is related to the tangential stretching SV at high latitudes in our radial field analysis. The other deep stretching mechanism found by Takahashi and 
Shimizu (2012) is associated with alternating flows in the direction perpendicular to columnar vortices, which is related to the radial stretching SV at low latitudes in our radial field analysis.

The significant contribution of radial stretching in the mid-shell roots indicates a possible underlying deep dynamo mechanism that sustains intense magnetic flux patches at the outer boundary. Because these high-latitude intense flux patches contribute significantly to the dominant geomagnetic axial dipole (Gubbins 1987; Olson and Amit 2006; Finlay 2008), radial stretching SV at depth may be considered as a mechanism to sustain the dipole.

\section{Abbreviations}

CMB: Core-mantle boundary; HN: Intense high-latitude normal polarity magnetic flux patch; HR: Intense high-latitude reversed polarity magnetic flux patch; LN: Intense low-latitude normal polarity magnetic flux patch; LR: Intense low-latitude reversed polarity magnetic flux patch; SV: Secular variation

\section{Acknowledgements}

We are grateful to two anonymous reviewers for their important comments. This work was partly carried out during several visits by D.P. to LPG Nantes.

\section{Funding}

This study was supported by the Centre National d'Etudes Spatiales (CNES) and the Observatório Nacional (ON/MCTI). D.P. was supported by a Ph.D. research grant from Coordenação de Aperfeiçoamento de Pessoal de Nível Superior (CAPES) and a grant by LPG-Nantes. K.P. was supported by la Région des Pays de la Loire and Coordenação de Aperfeiçoamento de Pessoal de Nível Superior (CAPES- Proc no BEX 2498/13-8) and Fundação de Amparo à Pesquisa do Estado do Rio de Janeiro (FAPERJ- E06/2015). This work acknowledges financial support from Région Pays de la Loire, project GeoPlaNet (convention no. 2016-10982).

\section{Availability of data and materials}

Data is not applicable. All material is available by request from Diego Peña (dpena@on.br).

\section{Authors' contributions}

HA ran the dynamo simulations. DP analyzed the models' output, produced the graphics, calculated the statistics, and wrote the paper. All authors read and improved the text and approved the final manuscript.

\section{Competing interests}

The authors declare that they have no competing interests.

\section{Publisher's Note}

Springer Nature remains neutral with regard to jurisdictional claims in published maps and institutional affiliations.

\section{Author details}

${ }^{1}$ Geophysics Department, Observatório Nacional, Rio de Janeiro 20921-400, Brazil. ${ }^{2}$ CNRS, Université de Nantes, Nantes Atlantiques Universités, UMR CNRS 6112, Laboratoire de Planétologie et de Géodynamique, 2 rue de la Houssinière, 44000 Nantes, France.

Received: 11 May 2017 Accepted: 22 December 2017

Published online: 05 February 2018

\section{References}

Amit H (2014) Can downwelling at the top of the Earth's core be detected in the geomagnetic secular variation? Phys Earth Planet Int 229:1 10-121

Amit $\mathrm{H}$, Olson P (2004) Helical core flow from geomagnetic secular variation. Phys Earth Planet Int 147:1-25

Amit $\mathrm{H}$, Olson P, Christensen UR (2007) Tests of core flow imaging methods with numerical dynamos. Geophys J Int 168:27-39
Anderson BJ, Johnson CL, Korth H, Purucker ME, Winslow RM, Slavin JA, Solomon SC, McNutt RL, Raines JM, Zurbuchen TH (2011) The global magnetic field of Mercury from MESSENGER orbital observations. Science 333(6051):1859-1862

Aubert J (2005) Steady zonal flows in spherical shell dynamos. J Fluid Mech 542:53-67

Aubert, J (2015) Geomagnetic forecasts driven by thermal wind dynamics in the Earth's core. Geophys J Int 203(3):1738-1751

Aubert J, Amit H, Hulot G, Olson P (2008a) Thermochemical flows couple the Earth's inner core growth to mantle heterogeneity. Nature 454(7205):758-761

Aubert J, Aurnou J, Wicht J (2008b) The magnetic structure of convection-driven numerical dynamos. Geophys J Int 172(3):945-956

Aubert J, Finlay CC, Fournier F (2013) Bottom up control of geomagnetic secular variation by the Earth's inner core. Nature 502:219-223

Aurnou J, Andreadis S, Zhu L, Olson P (2003) Experiments on convection in Earth's core tangent cylinder. Earth Planet Sci Lett 212(1): 119-134

Badro J, Siebert J, Nimmo F (2016) An early geodynamo driven by exsolution of mantle components from Earth's core. Nature 536:326-328

Bloxham J (1986) The expulsion of magnetic flux from the Earth's core. Geophys J R Astr Soc 87:669-678

Bloxham J, Jackson A (1991) Fluid flow near the surface of Earth's outer core. Rev Geophys 29(1):97-120

Christensen UR (2006) A deep dynamo generating Mercury's magnetic field. Nature 444:1056-1058

Christensen UR, Aubert J (2006) Scaling properties of convection-driven dynamos in rotating spherical shells and application to planetary magnetic fields. Geophys J Int 166:97-114

Christensen UR, Wicht J (2008) Models of magnetic field generation in partly stable planetary cores: Applications to Mercury and Saturn. Icarus 196:16-34

Christensen UR, Olson P, Glatzmaier G (1998) A dynamo model interpretation of geomagnetic field structures. Geophys Res Lett 25(10):1565-1568

Christensen UR, Aubert J, Hulot G (2010) Conditions for Earth-like geodynamo models. Earth Planet Sci Lett 296(3):487-496

Chulliat A, Hulot G, Newitt LR (2010) Magnetic flux expulsion from the core as a possible cause of the unusually large acceleration of the north magnetic pole during the 1990s. J Geophys Res 115(B7):1978-2012

Dziewonski AM, Anderson DL (1981) Preliminary reference Earth model. Phys Earth Planet Int 25(4):297-356

Finlay CC (2008) Historical variation of the geomagnetic axial dipole. Phys Earth Planet Int 170(1-2):1-14

Finlay CC, Amit H (2011) On flow magnitude and field flow alignment at Earth's core surface. Geophys J Int 186:175-192

Finlay CC, Jackson A (2003) Equatorially dominated magnetic field change at the surface of Earth's core. Science 300(5628):2084-2086

Gillet N, Pais MA, Jault D (2009) Ensemble inversion of time dependent core flow models. Geochem Geophys Geosyst 10(Q06004)

Gillet N, Schaeffer N, Jault D (2011) Rationale and geophysical evidence for quasi-geostrophic rapid dynamics within the Earth's outer core. Phys Earth Planet Int 187(3-4):380-390

Gillet N, Jault D, Finlay CC (2015) Planetary gyre, time-dependent eddies, torsional waves, and equatorial jets at the Earth's core surface. J Geophys Res Solid Earth 120(6):3991-4013

Gubbins D (1987) Mechanism for geomagnetic polarity reversals. Nature 326(6109):167-169

Gubbins D, Davies CJ (2013) The stratified layer at the core-mantle boundary caused by barodiffusion of oxygen, sulphur and silicon. Phys Earth Planet Int 215:21-28

Hartmann GA, Pacca IG (2009) Time evolution of the south atlantic magnetic anomaly. An Acad Bras Ciênc 81 (2):243-255

Helffrich G, Kaneshima S (2010) Outer-core compositional stratification from observed core wave speed profiles. Nature 468:807-810

Holme R (2015) Large-scale flow in the core. In: Schubert G (ed). Treatise on Geophysics, 2nd edn. Elsevier, Oxford. pp 91-113. chap 4

Jackson A, Jonkers A, Walker M (2000) Four centuries of geomagnetic secular variation from historical records. Phil Trans R Soc Lond A 358:957-990

Kageyama A, Sato T (1997a) Generation mechanism of a dipole field by a magnetohydrodynamic dynamo. Phys Rev E 55(4):4617-4626

Kageyama, A, Sato T (1997b) Velocity and magnetic field structures in a magnetohydrodynamic dynamo. Phys Plasmas 4(5):1569-1575 
de Koker N, Steinle-Neumann G, VIcek V (2012) Electrical resistivity and thermal conductivity of liquid Fe alloys at high P and T, and heat flux in Earth's core. Proc Natl Acad Sci USA 109(11):4070-4073

Konôpková Z, McWilliams S, Gómez-Pérez N, Goncharov A (2016) Direct measurement of thermal conductivity in solid iron at planetary core conditions. Nature 534:99-101

Kutzner C, Christensen UR (2002) From stable dipolar towards reversing numerical dynamos. Phys Earth Planet Int 131(1):29-45

Lesur V, Whaler K, Wardinski I (2015) Are geomagnetic data consistent with stably stratified flow at the core-mantle boundary? Geophys J Int 201:929-946

Livermore PW, Hollerbach R, Finlay CC (2017) An accelerating high-latitude jet in Earth's core. Nat Geosci 10:62-68. doi:10.1038/ngeo2859

Mininni PD (2011) Scale interactions in magnetohydrodynamic turbulence. Ann Rev Fluid Mech 43:377-397

Moffatt HK (1978) Magnetic field generation in electrically conducting fluids. Cambridge University Press, London

Ohta K, Kuwayama Y, Hirose K, Shimizu K, Ohishi Y (2016) Experimental determination of the electrical resistivity of iron at Earth's core conditions. Nature 534(7605):95-98

Oliveira JS, Langlais B, Pais MA, Amit H (2015) A modified equivalent source dipole method to model partially distributed magnetic field measurements, with application to Mercury. J Geophys Res 120:1075-1094

Olsen N, Mandea M (2008) Rapidly changing flows in the Earth's core. Nature Geosci 1(6):390-394

Olson P, Amit H (2006) Changes in Earth's dipole. Naturwissenschaften 93(11):519-542

Olson P, Aurnou J (1999) A polar vortex in the Earth's core. Nature 402(6758):170-173

Olson P, Christensen UR (2002) The time averaged magnetic field in numerical dynamos with non uniform boundary heat flow. Geophys J Int 151(3):809-823

Olson P, Christensen UR, Glatzmaier GA (1999) Numerical modeling of the geodynamo: mechanisms of field generation and equilibration. J Geophys Res 104(B5): 10,383-10,404

Olson P, Sumita I, Aurnou J (2002) Diffusive magnetic images of upwelling patterns in the core. J Geophys Res 107(B12):801-813

O'Rourke JG, Stevenson DJ (2016) Powering Earth's dynamo with magnesium precipitation from the core. Nature 529:387-389

Pais MA, Jault D (2008) Quasi-geostrophic flows responsible for the secular variation of the Earth's magnetic field. Geophys J Int 173(2):421-443

Peña D, Amit H, Pinheiro KJ (2016) Magnetic field stretching at the top of the shell of numerical dynamos. Earth Planets Space 68(1):1-21. doi:10.1186/s40623-016-0453-x

Pozzo M, Davies C, Gubbins D, Alfe D (2012) Thermal and electrical conductivity of iron at Earth's core conditions. Nature 485(7398):355-358

Press WH, Teukolsky SA, Vetterling WT, Flannery BP (1992) Numerical recipes in FORTRAN. Cambridge University Press, Cambridge

Takahashi F, Shimizu H (2012) A detailed analysis of a dynamo mechanism in a rapidly rotating spherical shell. J Fluid Mech 701:228-250

Tarduno J, Watkeys M, Huffman T, Cottrell R, Blackman E, Wendt A, Scribner C, Wagner C (2015) Antiquity of the South Atlantic Anomaly and evidence for top-down control on the geodynamo. Nat Commun 6:7865

Terra-Nova F, Amit H, Hartmann G, Trinidade RIF, Pinheiro KJ (2017) Relating the South Atlantic Anomaly and geomagnetic flux patches. Phys Earth Planet Int 266:39-53

Whaler K (1980) Does the whole of the Earth's core convect? Nature 287(5782):528-530

Wicht J (2002) Inner-core conductivity in numerical dynamo simulations. Phys Earth Planet Inter 132:281-302

Wicht J, Heyner D (2014) Mercury's magnetic field in the messenger era. In: Jin S (ed). Planetary Geodesy and Remote Sensing. CRC Press. pp 223-262

\section{Submit your manuscript to a SpringerOpen ${ }^{\circ}$ journal and benefit from:}

- Convenient online submission

- Rigorous peer review

- Open access: articles freely available online

- High visibility within the field

- Retaining the copyright to your article

Submit your next manuscript at $\gg$ springeropen.com 